\title{
$f(R)$ gravity constrained by PPN parameters and stochastic background of gravitational waves
}

\author{
S. Capozziello ${ }^{1}$, M. De Laurentis ${ }^{21}$, S. Nojiri ${ }^{3}$, S. D. Odintsov ${ }^{4}$ \\ ${ }^{1}$ Dipartimento di Scienze fisiche, Università di Napoli " Federico II", INFN Sez. di Napoli, \\ Compl. Univ. di Monte S. Angelo, Edificio G, Via Cinthia, I-80126, Napoli, Italy \\ ${ }^{2}$ Politecnico di Torino and INFN Sez. di Torino, \\ Corso Duca degli Abruzzi 24, I-10129 Torino, Italy \\ ${ }^{3}$ Department of Physics, Nagoya University, Nagoya 464-8602, Japan \\ ${ }^{4}$ Institucio Catalana de Recerca $i$ Estudis Avancats (ICREA) and Institut de Ciencies de l Espai (IEEC-CSIC), \\ Campus UAB, Facultat de Ciencies, Torre C5-Par-2a pl, E-08193 Bellaterra (Barcelona), Spain.
}

(Dated: October 24, 2018)

\begin{abstract}
We analyze seven different viable $f(R)$-gravities towards the Solar System tests and stochastic gravitational waves background. The aim is to achieve experimental bounds for the theory at local and cosmological scales in order to select models capable of addressing the accelerating cosmological expansion without cosmological constant but evading the weak field constraints. Beside large scale structure and galactic dynamics, these bounds can be considered complimentary in order to select self-consistent theories of gravity working at the infrared limit. It is demonstrated that seven viable $f(R)$-gravities under consideration not only satisfy the local tests, but additionally, pass the above PPN-and stochastic gravitational waves bounds for large classes of parameters.
\end{abstract}

PACS numbers: 04.50.+h, 04.80.Cc, 98.80.-k, 11.25.-w, 95.36.+x

\section{INTRODUCTION}

The currently observed accelerated expansion of the Universe suggests that cosmic flow dynamics is dominated by some unknown form of dark energy characterized by a large negative pressure. This picture comes out when such a new ingredient, beside baryonic and dark matter, is considered as a source in the r.h.s. of the field equations. Essentially, it should be some form of un-clustered, non-zero vacuum energy which, together with (clustered) dark matter, should drive the global cosmic dynamics.

Among the proposals to explain the experimental situation, the "concordance model", addressed as $\Lambda \mathrm{CDM}$, gives a reliable snapshot of the today observed Universe according to the CMBR, LSS and SNeIa data, but presents dramatic shortcomings as the "coincidence and cosmological constant problems" which point out its inadequacy to fully trace back the cosmological dynamics [1].

On the other hand, alternative theories of gravity, extending in some way General Relativity (GR), allows to pursue a different approach giving rise to suitable cosmological models where a late-time accelerated expansion can be achieved in several ways. This viewpoint does not require to find out candidates for dark energy and dark matter at fundamental level (they have not been detected up to now), it takes into account only the "observed" ingredients (i.e. gravity, radiation and baryonic matter), but the 1.h.s. of the Einstein equations has to be modified. Despite of this modification, it could be in agreement with the spirit of GR since the only request is that the Hilbert-Einstein action should be generalized asking for a gravitational interaction acting, in principle, in different ways at different scales [2].

The idea that Einstein gravity should be extended or corrected at large scales (infrared limit) or at high energies (ultraviolet limit) is suggested by several theoretical and observational issues. Quantum field theory in curved spacetimes, as well as the low-energy limit of String/M theory, both imply semi-classical effective actions containing higher-order curvature invariants or scalar-tensor terms. In addition, GR has been definitely tested only at Solar System scales while it may show several shortcomings if checked at higher energies or larger scales. Besides, the Solar System experiments are, up to now, not so conclusive to state that the only viable theory of gravity is GR: for example, the limits on PPN parameters should be greatly improved to fully remove degeneracies [3].

Of course, modifying the gravitational action asks for several fundamental challenges. These models can exhibit instabilities [4] or ghost-like behavior [5], while, on the other hand, they have to be matched with observations and experiments in the appropriate low energy limit.

Despite of all these issues, in the last years, some interesting results have been achieved in the framework of the so called $f(R)$-gravity at cosmological, Galactic and Solar System scales. Here $f(R)$ is a general (analytic) function of the Ricci scalar $R$ (see Refs. [6, 7, 8] for review). 
For example, there exist cosmological solutions that give the accelerated expansion of the universe at late times $[9,10,11,12]$. In addition, it has been discovered that some stability conditions can lead to avoid ghost and tachyon solutions. Furthermore there exist viable $f(R)$ models which satisfy both background cosmological constraints and stability conditions $[13,14,16,17,18,19,20,21]$ and results have been achieved in order to place constraints on $f(R)$ cosmological models by CMBR anisotropies and galaxy power spectrum [22, 23, 24]. Moreover, some of such viable models lead to the unification of early-time inflation with late-time acceleration [19, 20, 21].

On the other hand, by considering $f(R)$-gravity in the low energy limit, it is possible to obtain corrected gravitational potentials capable of explaining the flat rotation curves of spiral galaxies or the dynamics of galaxy clusters without considering huge amounts of dark matter [25, 26, 27, 28, 29, 30].

Furthermore, several authors have dealt with the weak field limit of fourth order gravity, in particular considering the PPN limit [32, 33, 34, 35, 36, 37, 38, 44] and the spherically symmetric solutions [39, 40, 41, 43].

This great deal of work needs an essential issue to be pursued: we need to compare experiments and probes at local scales (e.g. Solar System) with experiments and probes at large scales (Galaxy, extragalactic scales, cosmology) in order to achieve self-consistent $f(R)$ models. Some work has been done in this direction (see e.g. [16]) but the large part of efforts has been devoted to address single data sets (observations at a given redshift) by a single model which, several time, is not working at other scales than the one considered. In particular, a given $f(R)$ model, evading Solar System tests, should be not simply extrapolated at extragalactic and cosmological scales only requiring accelerated cosmological solutions but it should be confronted with data and probes coming from cosmological observations. Reliable models are then those matching data at very different scales (and redshifts).

In order to constrain further viable $f(R)$-models, one could take into account also the stochastic background of gravitational waves $(\mathrm{GW})$ which, together with cosmic microwave background radiation (CMBR), would carry a huge amount of information on the early stages of the Universe evolution. In fact, if detected, such a background could constitute a further probe for these theories at very high red-shift [62]. On the other hand, a key role for the production and the detection of the relic gravitational radiation background is played by the adopted theory of gravity [45, 46]. This means that the effective theory of gravity should be probed at zero, intermediate and high redshifts to be consistent at all scales and not simply extrapolated up to the last scattering surface, as in the case of GR.

The aim of this paper is to discuss the PPN Solar-System constraints and the GW stochastic background considering some recently proposed $f(R)$ gravity models [13, 14, 16, 19, 20, 21] which satisfy both cosmological and stability conditions mentioned above. Using the definition of PPN-parameters $\gamma$ and $\beta$ in terms of $f(R)$-models [38] and the definition of scalar GWs [47], we compare and discuss if it is possible to search for parameter ranges of $f(R)$-models working at Solar System and GW stochastic background scale. This phenomenological approach is complementary to the one proposed, e.g. in [16, 24] where also galactic and cosmological scales have been considered to constraint the models.

The layout of the paper is the following. In Sec. II, we review the field equations of $f(R)$ gravity in the metric approach and their scalar-tensor representation, useful to compare the theory with observations. In Sec.III, we review and discuss some viable $f(R)$ models capable of satisfying both local gravity prescriptions as well as the observed cosmological behavior. In particular, we discuss their stability conditions and the field values which have to achieved to fulfill physical bounds. Sec. IV is devoted to derive the values of model parameters in agreement with the PPN experimental constraints while, in Sec. V, we deal with the constraints coming from the stochastic background of GWs. These latter ones have to be confronted with those coming from PPN parameterization. Discussion and conclusions are drawn in Sec. VI. As a general remark, we find out that bounds coming from the interferometric ground-based (VIRGO, LIGO) and space (LISA) experiments could constitute a further probe for $f(R)$ gravity if matched with bounds at other scales.

\section{II. $f(R)$ GRAVITY}

Let us start from the following action

$$
\mathcal{S}=\mathcal{S}_{g}+\mathcal{S}_{m}=\frac{1}{k^{2}} \int d^{4} x \sqrt{-g}\left[R+f(R)+\mathcal{L}_{m}\right]
$$

where we have considered the gravitational and matter contributions and $k^{2} \equiv 16 \pi G$. The non-linear $f(R)$ term has been put in evidence with respect to the standard Hilbert-Einstein term $R$ and $\mathcal{L}_{m}$ is the perfect-fluid matter Lagrangian. The field equations are

$$
\frac{1}{2} g_{\mu \nu} F(R)-R_{\mu \nu} F^{\prime}(R)-g_{\mu \nu} \square F^{\prime}(R)+\nabla_{\mu} \nabla_{\nu} F^{\prime}(R)=-\frac{k^{2}}{2} T_{\mu \nu}^{(m)} .
$$


Here $F(R)=R+f(R)$ and $T_{\mu \nu}^{(m)}$ is the matter energy-momentum tensor. By introducing the auxiliary field $A$, one can rewrite the gravitational part in the Action (11) as

$$
\mathcal{S}_{g}=\frac{1}{k^{2}} \int d^{4} x \sqrt{-g}\left\{\left(1+f^{\prime}(A)\right)(R-A)+A+f(A)\right\} .
$$

As it is clear from Eq.(3), if $F^{\prime}(R)=1+f^{\prime}(R)<0$, the coupling $k_{\text {eff }}^{2}=k^{2} / F^{\prime}(A)$ becomes negative and the theory enters the anti-gravity regime. Note that it is not the case for the standard GR.

Action (3) can be recast in a scalar-tensor form. By using the conformal scale transformation $g_{\mu \nu} \rightarrow e^{\sigma} g_{\mu \nu}$ with $\sigma=-\ln \left(1+f^{\prime}(A)\right)$, the action can be written in the Einstein frame as follows [].

$$
\mathcal{S}_{E}=\frac{1}{k^{2}} \int d^{4} x \sqrt{-g}\left(R-\frac{3}{2} g^{\rho \sigma} \partial_{\rho} \sigma \partial_{\sigma} \sigma-V(\sigma)\right)
$$

where

$$
V(\sigma)=e^{\sigma} g\left(e^{-\sigma}\right)-e^{2 \sigma} f\left(g\left(e^{-\sigma}\right)\right)=\frac{A}{F^{\prime}(A)}-\frac{F(A)}{F^{\prime}(A)^{2}} .
$$

The form of $g\left(e^{-\sigma}\right)$ is given by solving $\sigma=-\ln \left(1+f^{\prime}(A)\right)=\ln F^{\prime}(A)$ as $A=g\left(e^{-\sigma}\right)$. The transformation $g_{\mu \nu} \rightarrow e^{\sigma} g_{\mu \nu}$ induces a coupling of the scalar field $\sigma$ with matter.

In general, an effective mass for $\sigma$ is defined as [21]

$$
m_{\sigma}^{2} \equiv \frac{1}{2} \frac{d^{2} V(\sigma)}{d \sigma^{2}}=\frac{1}{2}\left[\frac{A}{F^{\prime}(A)}-\frac{4 F(A)}{\left(F^{\prime}(A)\right)^{2}}+\frac{1}{F^{\prime \prime}(A)}\right]
$$

which, in the weak field limit, could induce corrections to the Newton law. This allows, as it is well known, to deal with the extra degrees of freedom of $f(R)$-gravity as an effective scalar field which reveals particularly useful in considering "chameleon" models [15]. This "parameterization" will be particularly useful to deal with the scalar component of GWs.

\section{III. $f(R)$ VIABLE MODELS}

Let us consider now a class of $f(R)$ models which do not contain cosmological constant and are explicitly designed to satisfy cosmological and Solar-System constraints in given limits of the parameter space. In practice, we choose a class of functional forms of $f(R)$ capable of matching, in principle, observational data (see [11] for the general approach). Firstly, the cosmological model should reproduce the CMBR constraints in the high-redshift regime (which agree with the presence of an effective cosmological constant). Secondly, it should give rise to an accelerated expansion, at low redshift, according to the $\Lambda \mathrm{CDM}$ model. Thirdly, there should be sufficient degrees of freedom in the parameterization to encompass low redshift phenomena (e.g. the large scale structure) according to the observations [24]. Finally, small deviations from GR should be consistent with Solar System tests. All these requirements suggest that we can assume the limits

$$
\begin{gathered}
\lim _{R \rightarrow \infty} f(R)=\text { constant, } \\
\lim _{R \rightarrow 0} f(R)=0,
\end{gathered}
$$

which are satisfied by a general class of broken power law models, proposed in [16], which are

$$
f_{I}(R)=-m^{2} \frac{c_{1}\left(\frac{R}{m^{2}}\right)^{n}}{c_{2}\left(\frac{R}{m^{2}}\right)^{n}+1}
$$

or otherwise written as 


$$
F_{I}(R)=R-\lambda R_{c} \frac{\left(\frac{R}{R_{c}}\right)^{2 n}}{\left(\frac{R}{R_{c}}\right)^{2 n}+1}
$$

where $m$ is a mass scale and $c_{1,2}$ are dimensionless parameters.

Besides, another viable class of models was proposed in [13]

$$
F_{I I}(R)=R+\lambda R_{c}\left[\left(1+\frac{R^{2}}{R_{c}^{2}}\right)^{-p}-1\right] .
$$

Since $F(R=0)=0$, the cosmological constant has to disappear in a flat spacetime. The parameters $\left\{n, p, \lambda, R_{c}\right\}$ are constants which should be determined by experimental bounds.

Other interesting models with similar features have been studied in [17, 18, 19, 20, 21]. In all these models, a de-Sitter stability point, responsible for the late-time acceleration, exists for $R=R_{1}(>0)$, where $R_{1}$ is derived by solving the equation $R_{1} f_{, R}\left(R_{1}\right)=2 f\left(R_{1}\right)$ [42]. For example, in the model (11), we have $R_{1} / R_{c}=3.38$ for $\lambda=2$ and $p=1$. If $\lambda$ is of the unit order, $R_{1}$ is of the same order of $R_{c}$. The stability conditions, $f_{, R}>0$ and $f_{, R R}>0$, are fulfilled for $R>R_{1}[13,18]$. Moreover the models satisfy the conditions for the cosmological viability that gives rise to the sequence of radiation, matter and accelerated epochs [18].

In the region $R \gg R_{c}$ both classes of models (9) and (11) behave as

$$
F_{I I I}(R) \simeq R-\lambda R_{c}\left[1-\left(R_{c} / R\right)^{2 s}\right]
$$

where $s$ is a positive constant. The model approaches $\Lambda$ CDM in the limit $R / R_{c} \rightarrow \infty$.

Finally, let also consider the class of models [14, 23, 31]

$$
F_{I V}(R)=R-\lambda R_{c}\left(\frac{R}{R_{c}}\right)^{q}
$$

Also in this case $\lambda, q$ and $R_{c}$ are positive constants (note that $n, p, s$ and $q$ have to converge toward the same values to match the observations). We do not consider the models whit negative $q$, because they suffer for instability problems associated with negative $F_{, R R}\left[22\right.$, , 48]. In Fig.(11), we have plotted some of the selected models as function of $\frac{R}{R_{c}}$ for suitable values of $\{p, n, q, s, \lambda\}$.

Let us now estimate $m_{\sigma}$ for the models discussed above. For Model I [16], when the curvature is large, we find

$$
f_{I}(R) \sim-\frac{m^{2} c_{1}}{c_{2}}+\frac{m^{2+2 n} c_{1}}{c_{2}^{2} R^{n}}+\cdots,
$$

and obtain the following expression:

$$
m_{\sigma}^{2} \sim \frac{m^{2} c_{2}^{2}}{2 n(n+1) c_{1}}\left(\frac{R}{m^{2}}\right)^{n+2}
$$

Here the order of the mass-dimensional parameter $m^{2}$ should be $m^{2} \sim 10^{-64} \mathrm{eV}^{2}$. Then in Solar System, where $R \sim 10^{-61} \mathrm{eV}^{2}$, the mass is given by $m_{\sigma}^{2} \sim 10^{-58+3 n} \mathrm{eV}^{2}$ while on the Earth atmosphere, where $R \sim 10^{-50} \mathrm{eV}^{2}$, it has to be $m_{\sigma}^{2} \sim 10^{-36+14 n} \mathrm{eV}^{2}$. The order of the radius of the Earth is $10^{7} \mathrm{~m} \sim\left(10^{-14} \mathrm{eV}\right)^{-1}$. Therefore the scalar field $\sigma$ is enough heavy if $n \gg 1$ and the correction to the Newton law is not observed, being extremely small. In fact, if we choose $n=10$, the order of the Compton length of the scalar field $\sigma$ becomes that of the Earth radius. On the other hand, in the Earth atmosphere, if we choose $n=10$, for example, we find that the mass is extremely large:

$$
m_{\sigma} \sim 10^{43} \mathrm{GeV} \sim 10^{29} \times M_{\text {Planck }} .
$$

Here $M_{\text {Planck }}$ is the Planck mass. Hence, the Newton law correction should be extremely small. 


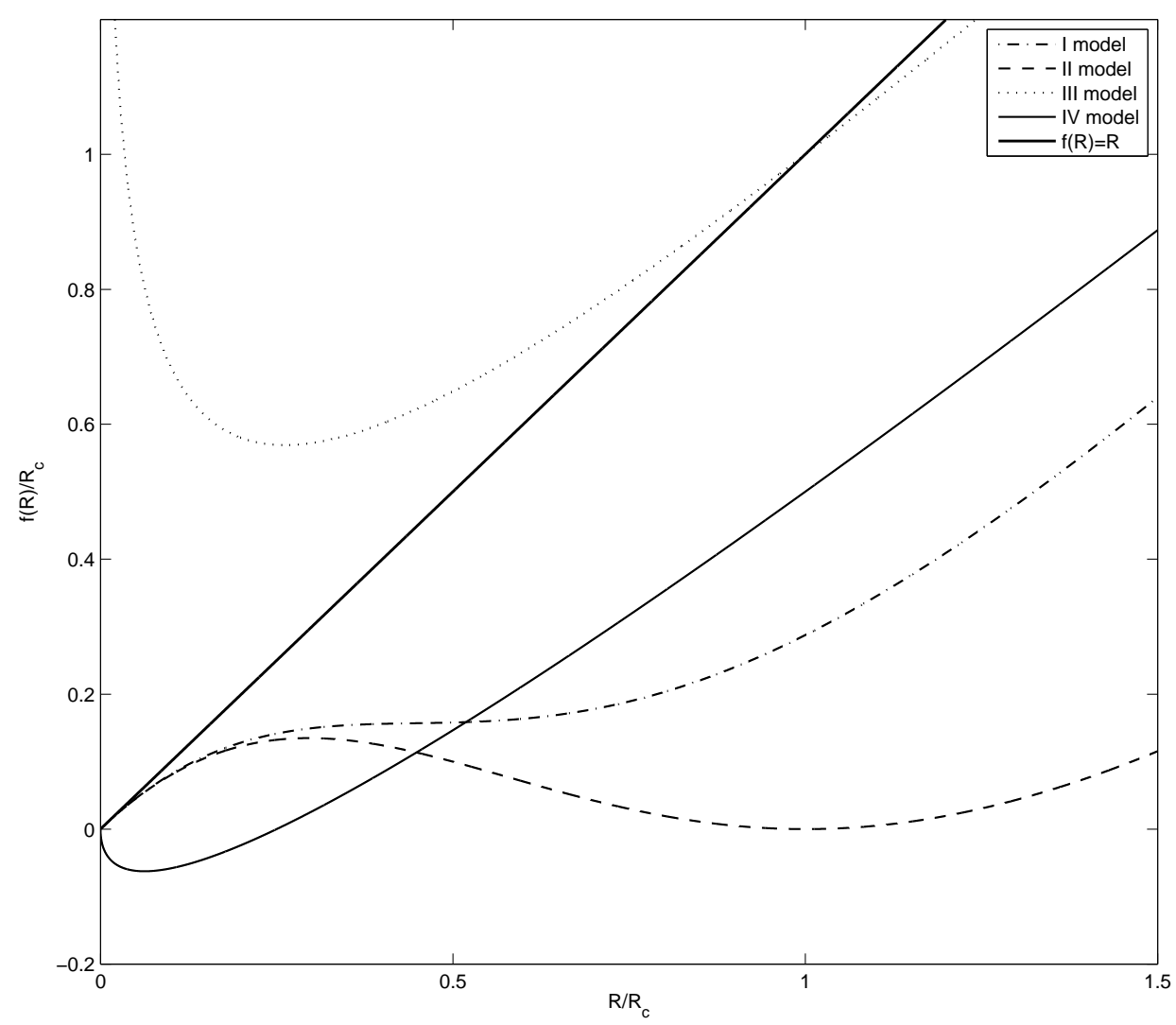

Figure 1: Plots of four different $F(R)$ models as function of $\frac{R}{R_{c}}$. Model I in Eq. (9) with $n=1$ and $\lambda=2$ (dashed line). Model II in Eq.(11) with $p=2, \lambda=0.95$ (dashdot line). Model III in Eq.(12) with $s=0.5$ and $\lambda=1.5$ (dotted). Model IV in Eq.(13) with $q=0.5$ and $\lambda=0.5$ (solid line). We also plot $F(R)=R$ (solid thick line) to see whether or not the stability condition $F_{, R}>0$ is violated.

In Model II

$$
f_{I I}(R)=-\lambda R_{0}\left[1-\left(1+\frac{R^{2}}{R_{0}^{2}}\right)^{-p}\right],
$$

if $R$ is large compared with $R_{0}$, whose order of magnitude is that of the curvature in the present universe, we find

$$
f_{I I}(R)=-\lambda R_{0}+\lambda \frac{R_{0}^{2 p+1}}{R^{2 p}}+\cdots
$$

By comparing Eq.(18) with Eq. (14), if the curvature is large enough when compared with $R_{0}$ or $m^{2}$, as in the Solar System or on the Earth, we can set the following identifications:

$$
\lambda R_{0} \leftrightarrow \frac{m^{2} c_{1}}{c_{2}}, \quad \lambda R_{0}^{2 p+1} \leftrightarrow \frac{m^{2+2 n} c_{1}}{c_{2}^{2}}, \quad 2 p \leftrightarrow n .
$$

We have $41 m^{2} \sim R_{0}$. Then, if $p$ is large enough, there is no correction to the Newton law as in Model I given by Eq.(10).

Let us now discuss the instability of fluid matter proposed in [48], which may appear if the matter-energy density (or the scalar curvature) is large enough when compared with the average density the Universe, as it is inside the Earth. Considering the trace of the above field equations and with a little algebra, one obtains

$$
\square R+\frac{F^{(3)}(R)}{F^{(2)}(R)} \nabla_{\rho} R \nabla^{\rho} R+\frac{F^{\prime}(R) R}{3 F^{(2)}(R)}-\frac{2 F(R)}{3 F^{(2)}(R)}=\frac{\kappa^{2}}{6 F^{(2)}(R)} T .
$$


Here $T$ is the trace of the matter energy-momentum tensor: $T \equiv T_{\rho}^{(m) \rho}$. We also denote the derivative $d^{n} F(R) / d R^{n}$ by $F^{(n)}(R)$. Let us now consider the perturbation of the Einstein gravity solutions. We denote the scalar curvature, given by the matter density in the Einstein gravity, by $R_{b} \sim\left(\kappa^{2} / 2\right) \rho>0$ and separate the scalar curvature $R$ into the sum of $R_{b}$ (background) and the perturbed part $R_{p}$ as $R=R_{b}+R_{p}\left(\left|R_{p}\right| \ll\left|R_{b}\right|\right)$. Then Eq.(20) leads to the perturbed equation:

$$
\begin{aligned}
0= & \square R_{b}+\frac{F^{(3)}\left(R_{b}\right)}{F^{(2)}\left(R_{b}\right)} \nabla_{\rho} R_{b} \nabla^{\rho} R_{b}+\frac{F^{\prime}\left(R_{b}\right) R_{b}}{3 F^{(2)}\left(R_{b}\right)} \\
& -\frac{2 F\left(R_{b}\right)}{3 F^{(2)}\left(R_{b}\right)}-\frac{R_{b}}{3 F^{(2)}\left(R_{b}\right)}+\square R_{p}+2 \frac{F^{(3)}\left(R_{b}\right)}{F^{(2)}\left(R_{b}\right)} \nabla_{\rho} R_{b} \nabla^{\rho} R_{p}+U\left(R_{b}\right) R_{p} .
\end{aligned}
$$

Here the potential $U\left(R_{b}\right)$ is given by

$$
\begin{aligned}
U\left(R_{b}\right) \equiv & \left(\frac{F^{(4)}\left(R_{b}\right)}{F^{(2)}\left(R_{b}\right)}-\frac{F^{(3)}\left(R_{b}\right)^{2}}{F^{(2)}\left(R_{b}\right)^{2}}\right) \nabla_{\rho} R_{b} \nabla^{\rho} R_{b}+\frac{R_{b}}{3} \\
& -\frac{F^{(1)}\left(R_{b}\right) F^{(3)}\left(R_{b}\right) R_{b}}{3 F^{(2)}\left(R_{b}\right)^{2}}-\frac{F^{(1)}\left(R_{b}\right)}{3 F^{(2)}\left(R_{b}\right)}+\frac{2 F\left(R_{b}\right) F^{(3)}\left(R_{b}\right)}{3 F^{(2)}\left(R_{b}\right)^{2}}-\frac{F^{(3)}\left(R_{b}\right) R_{b}}{3 F^{(2)}\left(R_{b}\right)^{2}}
\end{aligned}
$$

It is convenient to consider the case where $R_{b}$ and $R_{p}$ are uniform and do not depend on the spatial coordinates. Hence, the d'Alembert operator can be replaced by the second derivative with respect to the time, that is: $\square R_{p} \rightarrow-\partial_{t}^{2} R_{p}$. Eq.(22) assumes the following structure:

$$
0=-\partial_{t}^{2} R_{p}+U\left(R_{b}\right) R_{p}+\text { const }
$$

If $U\left(R_{b}\right)>0, R_{p}$ becomes exponentially large with time, i.e. $R_{p} \sim \mathrm{e}^{\sqrt{U\left(R_{b}\right)} t}$, and the system becomes unstable.

In the $1 / R$-model, considering the background values, we find

$$
\begin{aligned}
& U\left(R_{b}\right)=-R_{b}+\frac{R_{b}^{3}}{6 \mu^{4}} \sim \frac{R_{0}^{3}}{\mu^{4}} \sim\left(10^{-26} \mathrm{sec}\right)^{-2}\left(\frac{\rho_{m}}{\mathrm{~g} \mathrm{~cm}^{-3}}\right)^{3}, \\
& R_{b} \sim\left(10^{3} \mathrm{sec}\right)^{-2}\left(\frac{\rho_{m}}{\mathrm{~g} \mathrm{~cm}^{-3}}\right) .
\end{aligned}
$$

Here the mass parameter $\mu$ is of the order

$$
\mu^{-1} \sim 10^{18} \sec \sim\left(10^{-33} \mathrm{eV}\right)^{-1}
$$

Eq.(24) tells us that the model is unstable and it would decay in $10^{-26} \mathrm{sec}$ (considering the Earth size). In Model I, however, $U\left(R_{b}\right)$ is negative:

$$
U\left(R_{0}\right) \sim-\frac{(n+2) m^{2} c_{2}^{2}}{c_{1} n(n+1)}<0
$$

Therefore, there is no matter instability.

For Model (17), as it is clear from the identifications (19), there is no matter instability too.

In order to study the stability of the de Sitter solution, let us proceed as follows. From the field equations (2), we obtain the trace

$$
\square f^{\prime}(R)=\frac{1}{3}\left[R-f^{\prime}(R) R+2 f(R)+\kappa^{2} T\right]
$$

Here, as above, $F(R)$ is $F(R)=R+f(R)$ and $T \equiv g^{\mu \nu} T_{\mu \nu}^{(m)}$.

Now we consider the (in)stability around the de Sitter solution, where $R=R_{0}$, and therefore $f\left(R_{0}\right)$ and $f^{\prime}\left(R_{0}\right)$, are constants. Then since the 1.h.s. in Eq.(27) vanishes for $R=R_{0}$, we find

$$
R_{0}-f^{\prime}\left(R_{0}\right) R_{0}+2 f\left(R_{0}\right)+\kappa^{2} T_{0}=0
$$

Let us expand both sides of (28) around $R=R_{0}$ as

$$
R=R_{0}+\delta R
$$


One obtains

$$
f^{\prime \prime}\left(R_{0}\right) \square \delta R=\frac{1}{3}\left(1-f^{\prime \prime}\left(R_{0}\right) R_{0}+f^{\prime}\left(R_{0}\right)\right) \delta R .
$$

Since

$$
\square \delta R=-\frac{d^{2} \delta R}{d t^{2}}-3 H_{0} \frac{d \delta R}{d t}
$$

in the de Sitter background, if

$$
C\left(R_{0}\right) \equiv \lim _{R \rightarrow R_{0}} \frac{1-f^{\prime \prime}(R) R+f^{\prime}(R)}{f^{\prime \prime}(R)}>0,
$$

the de Sitter background is stable but, if $C\left(R_{0}\right)<0$, the de Sitter background is unstable. The expression for $C\left(R_{0}\right)$ could be valid even if $f^{\prime \prime}\left(R_{0}\right)=0$. More precisely, the solution of (30) is given by

$$
\delta R=A_{+} \mathrm{e}^{\lambda_{+} t}+A_{-} \mathrm{e}^{\lambda_{-} t}
$$

Here $A_{ \pm}$are constants and

$$
\lambda_{ \pm}=\frac{-3 H_{0} \pm \sqrt{9 H_{0}^{2}-C\left(R_{0}\right)}}{2} .
$$

Then, if $C\left(R_{0}\right)<0, \lambda_{+}$is always positive and the perturbation grows up. This leads to the instability. We have also to note that, when $C\left(R_{0}\right)$ is positive, if $C\left(R_{0}\right)>9 H_{0}^{2}, \delta R$ oscillates and the amplitude becomes exponentially small being:

$$
\delta R=\left(A \cos \omega_{0} t+B \sin \omega_{0} t\right) \mathrm{e}^{-3 H_{0} t / 2}, \quad \omega \equiv \frac{\sqrt{C\left(R_{0}\right)-9 H_{0}^{2}}}{2} .
$$

Here $A$ and $B$ are constant. On the other hand, if $C\left(R_{0}\right)<9 H_{0}^{2}$, there is no oscillation in $\delta R$.

Let us now consider the case where the matter contribution $T$ can be neglected in the de Sitter background and assume $f^{\prime}(R)=0$ in the same background. We can assume that there are two de Sitter background solutions satisfying $f^{\prime}(R)=0$, for $R=R_{1}$ and $R=R_{2}$ as it could be the physical case if one asks for an inflationary and a dark energy epoch. We also assume $f^{\prime}(R) \neq 0$ if $R_{1}<R<R_{2}$ or $R_{2}<R<R_{1}$. In the case $C\left(R_{1}\right)<0$ and $C\left(R_{2}\right)>0$, the de Sitter solution, corresponding to $R=R_{1}$, is unstable but the solution corresponding to $R=R_{2}$ is stable. Then there should be a solution where the (nearly) de Sitter solution corresponding to $R_{1}$ transits to the (nearly) de Sitter solution $R_{2}$. Since the solution corresponding to $R_{2}$ is stable, the universe remains in the de Sitter solution corresponding to $R_{2}$ and there is no more transition to any other de Sitter solution.

As an example, we consider Model I. For large curvature values, we find

$$
f_{\mathrm{I}}(R)=-\Lambda+\frac{\alpha}{R^{2 n+1}} .
$$

Here $\Lambda$ and $\alpha$ are positive constants and $n$ is a positive integer. Then we find

$$
C(R) \sim \frac{1}{f^{\prime \prime}(R)} \sim \frac{R^{2 n+2}}{2 n(2 n+1) \alpha}>0 .
$$

This means that the de Sitter solution in Model I can be stable. We have also to note that $C\left(R_{0}\right) \sim H_{0}^{4 n+4} / m^{4 n+2}$. Here $m^{2}$ is the mass scale introduced in [16] and $m^{2} \ll H_{0}^{2}$ : this means that $C\left(R_{0}\right) \gg 9 H_{0}^{2}$ and therefore there could be no oscillation.

We may also consider the model proposed in [19](here Model V):

$$
f_{V}(R)=\frac{\alpha R^{2 n}-\beta R^{n}}{1+\gamma R^{n}}
$$

Here $\alpha, \beta$, and $\gamma$ are positive constants and $n$ is a positive integer. In Fig 2 , we show the behavior of Model $\mathrm{V}$ and of its first derivative. When the curvature is large $(R \rightarrow \infty), f(R)$ behaves as a power law. Since the derivative of $f(R)$ is given by

$$
f_{V}^{\prime}(R)=\frac{n R^{n-1}\left(\alpha \gamma R^{2 n}-2 \alpha R^{n}-\beta\right)}{\left(1+\gamma R^{n}\right)^{2}}
$$


we find that the curvature $R_{0}$ in the present universe, which satisfies the condition $f^{\prime}\left(R_{0}\right)=0$, is given by

$$
R_{0}=\left[\frac{1}{\gamma}\left(1+\sqrt{1+\frac{\beta \gamma}{\alpha}}\right)\right]^{1 / n}
$$

and

$$
f\left(R_{0}\right) \sim-2 \tilde{R}_{0}=\frac{\alpha}{\gamma^{2}}\left(1+\frac{(1-\beta \gamma / \alpha) \sqrt{1+\beta \gamma / \alpha}}{2+\sqrt{1+\beta \gamma / \alpha}}\right) .
$$

As shown in [19], the magnitudes of the parameters is given by

$$
\alpha \sim 2 \tilde{R}_{0} R_{0}^{-2 n}, \beta \sim 4 \tilde{R}_{0}^{2} R_{0}^{-2 n} R_{I}^{n-1}, \gamma \sim 2 \tilde{R}_{0} R_{0}^{-2 n} R_{I}^{n-1} .
$$

Here $R_{I}$ is the curvature in the inflationary epoch and we have assumed $f\left(R_{I}\right) \sim(\alpha / \gamma) R_{I}^{n} \sim R_{I}$.

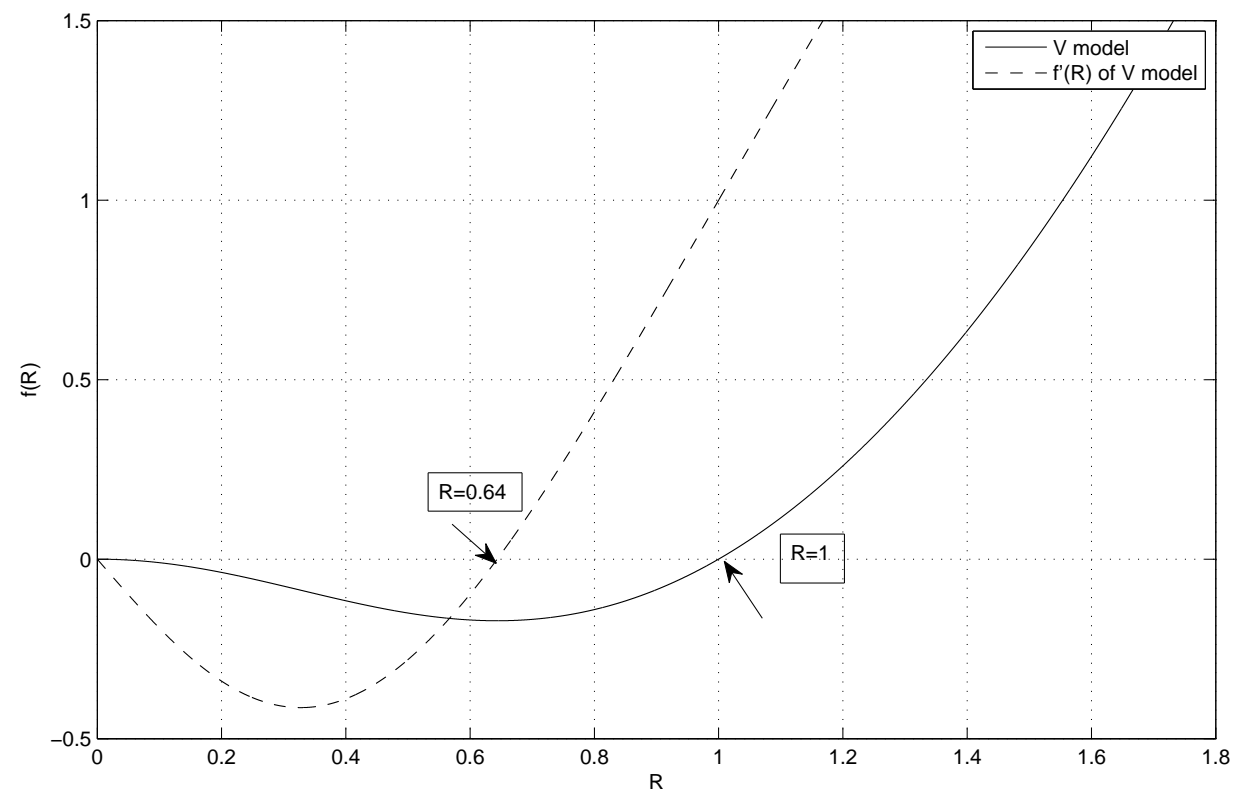

Figure 2: Plots of Model V (38) (solid line) and its first derivative (dashed line). Here $n=2$ and $\alpha, \beta, \gamma$ are assumed as in (42) with the value of $R_{0}$ taken in the Solar System. $f^{\prime}(R)$ is negative for $0<R<0.64 . f(R)$ is given in the range $0<R<1$ where we have adopted suitable units.

$C\left(R_{0}\right)$ in (32) is given by

$$
C\left(R_{0}\right) \sim \frac{1}{f^{\prime \prime}\left(R_{0}\right)}=\frac{1+\gamma R_{0}^{n}}{2 n^{2} \alpha R_{0}^{2 n-2}\left(\gamma R_{0}^{n}-1\right)} .
$$

By using the relations (42), we find

$$
C\left(R_{0}\right) \sim \frac{R_{0}^{2}}{4 n^{2} \tilde{R}_{0}}
$$

which is positive and therefore the de Sitter solution is stable. We notice that $C\left(R_{0}\right)<9 H_{0}^{2}$ and therefore, there could occur oscillations as in (35).

Furthermore, we can take into account the following model [20] (Model VI):

$$
f_{V I}(R)=-\alpha\left[\tanh \left(\frac{b\left(R-R_{0}\right)}{2}\right)+\tanh \left(\frac{b R_{0}}{2}\right)\right]=-\alpha\left[\frac{\mathrm{e}^{b\left(R-R_{0}\right)}-1}{\mathrm{e}^{b\left(R-R_{0}\right)}+1}+\frac{\mathrm{e}^{b R_{0}}-1}{\mathrm{e}^{b R_{0}}+1}\right]
$$


where $\alpha$ and $b$ are positive constants. When $R \rightarrow 0$, we find that

$$
f_{V I}(R) \rightarrow-\frac{\alpha b R}{2 \cosh ^{2}\left(\frac{b R_{0}}{2}\right)},
$$

and thus $f(0)=0$. On the other hand, when $R \rightarrow+\infty$,

$$
f_{V I}(R) \rightarrow-2 \Lambda_{\text {eff }} \equiv-\alpha\left[1+\tanh \left(\frac{b R_{0}}{2}\right)\right] .
$$

If $R \gg R_{0}$, in the present universe, $\Lambda_{\text {eff }}$ plays the role of the effective cosmological constant. We also obtain

$$
f_{V I}^{\prime}(R)=-\frac{\alpha b}{2 \cosh ^{2}\left(\frac{b\left(R-R_{0}\right)}{2}\right)},
$$

which has a minimum when $R=R_{0}$, that is:

$$
f_{V I}^{\prime}\left(R_{0}\right)=-\frac{\alpha b}{2}
$$

Then in order to avoid anti-gravity, we find

$$
0<1+f_{V I}^{\prime}\left(R_{0}\right)=1-\frac{\alpha b}{2} .
$$

Beside the above model, we can consider a model which is able to describe, in principle, both the early inflation and the late acceleration epochs. The following two-step model [20] (Model VII):

$$
f_{V I I}(R)=-\alpha_{0}\left[\tanh \left(\frac{b_{0}\left(R-R_{0}\right)}{2}\right)+\tanh \left(\frac{b_{0} R_{0}}{2}\right)\right]-\alpha_{I}\left[\tanh \left(\frac{b_{I}\left(R-R_{I}\right)}{2}\right)+\tanh \left(\frac{b_{I} R_{I}}{2}\right)\right],
$$

could be useful to this goal. Let us assume

$$
R_{I} \gg R_{0}, \quad \alpha_{I} \gg \alpha_{0}, \quad b_{I} \ll b_{0},
$$

and

$$
b_{I} R_{I} \gg 1
$$

When $R \rightarrow 0$ or $R \ll R_{0} \ll R_{I}, f_{V I I}(R)$ behaves as

$$
f_{V I I}(R) \rightarrow-\left[\frac{\alpha_{0} b_{0}}{2 \cosh ^{2}\left(\frac{b_{0} R_{0}}{2}\right)}+\frac{\alpha_{I} b_{I}}{2 \cosh ^{2}\left(\frac{b_{I} R_{I}}{2}\right)}\right] R,
$$

and we find again $f_{V I I}(0)=0$. When $R \gg R_{I}$, we find

$$
f(R)_{V I I} \rightarrow-2 \Lambda_{I} \equiv-\alpha_{0}\left[1+\tanh \left(\frac{b_{0} R_{0}}{2}\right)\right]-\alpha_{I}\left[1+\tanh \left(\frac{b_{I} R_{I}}{2}\right)\right] \sim-\alpha_{I}\left[1+\tanh \left(\frac{b_{I} R_{I}}{2}\right)\right] .
$$

On the other hand, when $R_{0} \ll R \ll R_{I}$, we find

$$
f_{V I I}(R) \rightarrow-\alpha_{0}\left[1+\tanh \left(\frac{b_{0} R_{0}}{2}\right)\right]-\frac{\alpha_{I} b_{I} R}{2 \cosh ^{2}\left(\frac{b_{I} R_{I}}{2}\right)} \sim-2 \Lambda_{0} \equiv-\alpha_{0}\left[1+\tanh \left(\frac{b_{0} R_{0}}{2}\right)\right] .
$$

Here, we have assumed the condition (53). We also find

$$
f_{V I I}^{\prime}(R)=-\frac{\alpha_{0} b_{0}}{2 \cosh ^{2}\left(\frac{b_{0}\left(R-R_{0}\right)}{2}\right)}-\frac{\alpha_{I} b_{I}}{2 \cosh ^{2}\left(\frac{b_{I}\left(R-R_{I}\right)}{2}\right)},
$$

which has two minima for $R \sim R_{0}$ and $R \sim R_{I}$. When $R=R_{0}$, we obtain

$$
f_{V I I}^{\prime}\left(R_{0}\right)=-\alpha_{0} b_{0}-\frac{\alpha_{I} b_{I}}{2 \cosh ^{2}\left(\frac{b_{I}\left(R_{0}-R_{I}\right)}{2}\right)}>-\alpha_{I} b_{I}-\alpha_{0} b_{0} .
$$


On the other hand, when $R=R_{I}$, we get

$$
f_{V I I}^{\prime}\left(R_{I}\right)=-\alpha_{I} b_{I}-\frac{\alpha_{0} b_{0}}{2 \cosh ^{2}\left(\frac{b_{0}\left(R_{0}-R_{I}\right)}{2}\right)}>-\alpha_{I} b_{I}-\alpha_{0} b_{0} .
$$

Then, in order to avoid the anti-gravity behavior, we find

$$
\alpha_{I} b_{I}+\alpha_{0} b_{0}<1 .
$$

Let us now investigate the correction to the Newton potential and the matter instability issue related to Models VI and VII. In the Solar System domain, on or inside the Earth, where $R \gg R_{0}, f(R)$ in Eq.(45) can be approximated by

$$
f_{V I}(R) \sim-2 \Lambda_{\text {eff }}+2 \alpha \mathrm{e}^{-b\left(R-R_{0}\right)} .
$$

On the other hand, since $R_{0} \ll R \ll R_{I}$, by assuming Eq. (53), $f(R)$ in (51) can be also approximated by

$$
f_{V I I}(R) \sim-2 \Lambda_{0}+2 \alpha \mathrm{e}^{-b_{0}\left(R-R_{0}\right)},
$$

which has the same expression, after having identified $\Lambda_{0}=\Lambda_{\text {eff }}$ and $b_{0}=b$. Then, we may check the case of (61) only. In this case, the effective mass has the following form

$$
m_{\sigma}^{2} \sim \frac{\mathrm{e}^{b\left(R-R_{0}\right)}}{4 \alpha b^{2}}
$$

which could be again very large. In fact, in the Solar System, we find $R \sim 10^{-61} \mathrm{eV}^{2}$. Even if we choose $\alpha \sim$ $1 / b \sim R_{0} \sim\left(10^{-33} \mathrm{eV}\right)^{2}$, we find that $m_{\sigma}^{2} \sim 10^{1,000} \mathrm{eV}^{2}$, which is, ultimately, extremely heavy. Then, there will be no appreciable correction to the Newton law. In the Earth atmosphere, $R \sim 10^{-50} \mathrm{eV}^{2}$, and even if we choose $\alpha \sim 1 / b \sim R_{0} \sim\left(10^{-33} \mathrm{eV}\right)^{2}$ again, we find that $m_{\sigma}^{2} \sim 10^{10,000,000,000} \mathrm{eV}^{2}$. Then, a correction to the Newton law is never observed in such models. In this case, we find that the effective potential $U\left(R_{b}\right)$ has the form

$$
U\left(R_{e}\right)=-\frac{1}{2 \alpha b}\left(2 \Lambda+\frac{1}{b}\right) \mathrm{e}^{-b\left(R_{e}-R_{0}\right)},
$$

which could be negative, what would suppress any instability.

In order that a de Sitter solution exists in $f(R)$-gravity, the following condition has to be satisfied:

$$
R=R f^{\prime}(R)-2 f(R) .
$$

For the model (45), the r.h.s of (65) has the following form:

$$
R=-\frac{b \alpha R}{2 \cosh ^{2}\left(\frac{b\left(R-R_{0}\right)}{2}\right)}+2 \alpha\left[\tanh \left(\frac{b\left(R-R_{0}\right)}{2}\right)+\tanh \left(\frac{b R_{0}}{2}\right)\right] .
$$

For large $R$, the r.h.s. behaves as

$$
-\frac{b \alpha R}{2 \cosh ^{2}\left(\frac{b\left(R-R_{0}\right)}{2}\right)}+2 \alpha\left[\tanh \left(\frac{b\left(R-R_{0}\right)}{2}\right)+\tanh \left(\frac{b R_{0}}{2}\right)\right] \rightarrow 2 \alpha,
$$

although the l.h.s. goes to infinity. On the other hand, when $R$ is small, the r.h.s. behaves as

$$
-\frac{b \alpha R}{2 \cosh ^{2}\left(\frac{b\left(R-R_{0}\right)}{2}\right)}+2 \alpha\left[\tanh \left(\frac{b\left(R-R_{0}\right)}{2}\right)+\tanh \left(\frac{b R_{0}}{2}\right)\right] \rightarrow \frac{b \alpha R}{2 \cosh ^{2}\left(\frac{b R_{0}}{2}\right)} .
$$

Then if

$$
\frac{b \alpha}{2 \cosh ^{2}\left(\frac{b R_{0}}{2}\right)}>1
$$


there is a de Sitter solution. Combining Eq.(69) with Eq.(50), we find

$$
2>\alpha b>\frac{1}{2 \cosh ^{2}\left(\frac{b R_{0}}{2}\right)} .
$$

The stability, as above, is given by $C\left(R_{\mathrm{dS}}\right)$, where $R_{\mathrm{dS}}$ is the solution of (66). The expression is given by

$$
C\left(R_{\mathrm{dS}}\right)=-R_{\mathrm{dS}}+\frac{2 \cosh ^{3}\left(\frac{b\left(R_{\mathrm{dS}}-R_{0}\right)}{2}\right)}{\alpha b^{2} \sinh \left(\frac{b\left(R_{\mathrm{dS}}-R_{0}\right)}{2}\right)}-\frac{1}{b \tanh \left(\frac{b\left(R_{\mathrm{dS}}-R_{0}\right)}{2}\right)} .
$$

Let us now rewrite Eq.(66) as follows,

$$
R_{\mathrm{dS}}=2 \alpha\left[\tanh \left(\frac{b\left(R_{\mathrm{dS}}-R_{0}\right)}{2}\right)+\tanh \left(\frac{b R_{0}}{2}\right)\right]\left[1+\frac{\alpha b}{2 \cosh ^{2}\left(\frac{b\left(R_{\mathrm{dS}}-R_{0}\right)}{2}\right)}\right]^{-1} .
$$

Then by using (72), we may rewrite (71) in the following form:

$$
C\left(R_{\mathrm{dS}}\right)=\frac{-\alpha^{2} b^{2}\left(1-x^{2}\right)\left[\left(x-x_{0}\right)^{2}+1-x_{0}^{2}\right]+4}{\alpha b^{2} x\left(1-x^{2}\right)\left[2+\alpha b\left(1-x^{2}\right)\right]}
$$

where

$$
x=\tanh \left(\frac{b\left(R_{\mathrm{dS}}-R_{0}\right)}{2}\right), \quad x_{0}=-\tanh \left(\frac{b R_{0}}{2}\right)
$$

and therefore we have

$$
-1<x_{0} \leq x<1, \quad x_{0}<0 .
$$

Let us now consider (66) in order to find a de Sitter solution. Since Eq.(66) is difficult to solve in general, we assume $0<R_{\mathrm{dS}} \ll R_{0}$. Then we find

$$
R_{\mathrm{dS}}=\frac{\epsilon}{b x_{0}}, \quad \epsilon \equiv 1-\frac{2 \cosh ^{2}\left(\frac{b R_{0}}{2}\right)}{\alpha b}=1-\frac{2}{\alpha b\left(1-x_{0}^{2}\right)} .
$$

Eq.69) tells that the parameter $\epsilon$ is positive and, by assumption, very small: $0<\epsilon \ll 1$. Since $\epsilon$ is small, by using Eqs.(74), we find

$$
x=x_{0}+\frac{\left(1-x_{0}^{2}\right)}{2 x_{0}} \epsilon+\mathcal{O}\left(\epsilon^{2}\right) .
$$

Then by using the expression (73) for $C\left(R_{\mathrm{dS}}\right)$, we find

$$
C\left(R_{\mathrm{dS}}\right) \sim \frac{-\alpha^{2} b^{2}\left(1-x_{0}^{2}\right)^{2}+4}{\alpha b^{2} x_{0}\left(1-x_{0}^{2}\right)\left[2+\alpha b\left(1-x_{0}^{2}\right)\right]} .
$$

From the definition of $\epsilon$ in (76), we find

$$
\alpha b\left(1-x_{0}^{2}\right)=2+2 \epsilon+\mathcal{O}\left(\epsilon^{2}\right)
$$

and then, from Eq.(79), Eq.(78) can be written as follows;

$$
C\left(R_{\mathrm{dS}}\right) \sim-\frac{\epsilon}{b x_{0}}
$$

Since $x_{0}<0$ in the condition (75) , we find $C\left(R_{\mathrm{dS}}\right)>0$ and therefore the de Sitter solution is stable.

In Fig. 3, we have plotted the two models (45) and (51) written in the form $F(R)=R+f(R)$. We have used the inequalities (52) assuming, $R_{I} \sim \rho_{g} \sim 10^{-24} \mathrm{~g} / \mathrm{cm}^{3}$ for the Galactic density in the Solar vicinity and $R_{0} \sim \rho_{g} \sim 10^{-29} \mathrm{~g} / \mathrm{cm}^{3}$ for the present cosmological density. .

Our task is now to find reliable experimental bounds for such models working at small and large scales. To this goal, we shall take into account constraints coming from Solar System experiments (which, at present, are capable of giving upper limits on the PPN parameters) and constraints coming from interferometers, in particular those giving limits on the (eventual) scalar components of GWs. If constraints (and in particular the ranges of model parameters given by them) are comparable, this could constitute, besides other experimental and observational probes, a good hint to achieve a self-consistent $f(R)$ theory at very different scales. 


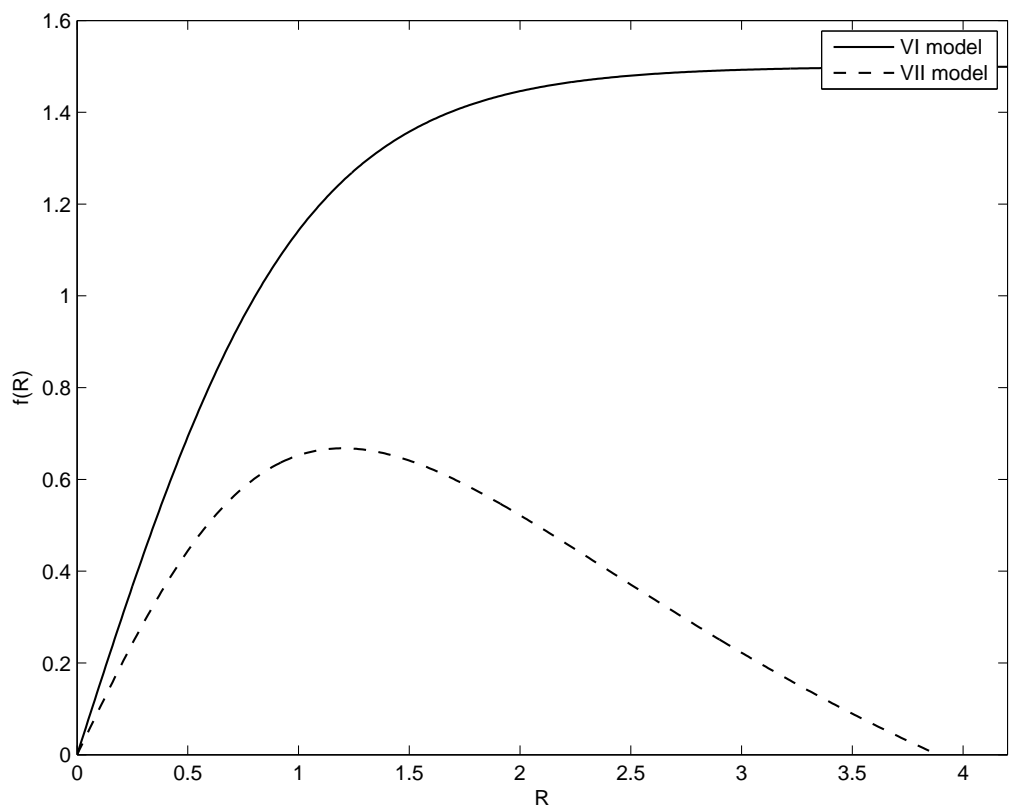

Figure 3: Plots of Model VI (45) (solid line) and Model VII (51) (dashed line). Here $b=2$ and $b_{I}=0.5$ with $\alpha=1.5$ and $\alpha_{I}=2$. The value of $R_{I}$ is taken in the Solar System while $R_{0}$ corresponds to the present cosmological value.

\begin{tabular}{|c|c|}
\hline Mercury perihelion Shift & $|2 \gamma-\beta-1|<3 \times 10^{-3}$ \\
\hline \hline Lunar Laser Ranging & $4 \beta-\gamma-3=(0.7 \pm 1) \times 10^{-3}$ \\
\hline Very Long Baseline Interferometer & $|\gamma-1|<4 \times 10^{-4}$ \\
\hline Cassini Spacecraft & $\gamma-1=(2.1 \pm 2.3) \times 10^{-5}$ \\
\hline
\end{tabular}

Table I: Solar System experimental constraints on the PPN parameters.

\section{CONSTRAINING $f(R)$-MODELS BY PPN PARAMETERS}

The above models can be constrained at Solar System level by considering the PPN formalism. This approach is extremely important in order to test gravitational theories and to compare them with GR. As it is shown in [33, 38], one can derive the PPN-parameters $\gamma$ and $\beta$ in terms of a generic analytic function $F(R)$ and its derivative

$$
\begin{gathered}
\gamma-1=-\frac{F^{\prime \prime}(R)^{2}}{F^{\prime}(R)+2 F^{\prime \prime}(R)^{2}}, \\
\beta-1=\frac{1}{4}\left[\frac{F^{\prime}(R) \cdot F^{\prime \prime}(R)}{2 F^{\prime}(R)+3 F^{\prime \prime}(R)^{2}}\right] \frac{d \gamma}{d R} .
\end{gathered}
$$

These quantities have to fulfill the constraints coming from the Solar System experimental tests summarized in Table I. They are the perihelion shift of Mercury [49], the Lunar Laser Ranging [50], the upper limits coming from the Very Long Baseline Interferometry (VLBI) [51] and the results obtained from the Cassini spacecraft mission in the delay of the radio waves transmission near the Solar conjunction [52].

Let us take into account before the $f(R)$-models (10)-13i). Specifically, we want to investigate the values or the ranges of parameters in which they match the Solar-System experimental constraints in Table I In other words, we 
use these models to search under what circumstances it is possible to significantly address cosmological observations by $f(R)$-gravity and, simultaneously, evade the local tests of gravity.

By integrating Eqs. (81)- (82), one obtains $f(R)$ solutions depending on $\beta$ and $\gamma$ which has to be confronted with $\beta_{\text {exp }}$ and $\gamma_{\text {exp }}$ [38]. If we plug into such equations the models (10)-(13) and the experimental values of PPN parameters, we will obtain algebraic constraints for the phenomenological parameters $\{n, p, q, \lambda, s\}$. This is the issue which we want to take into account in this section.

From Eq.(81), assuming $F^{\prime}(R)+2 F^{\prime \prime}(R)^{2} \neq 0$ and defining $A=\left|\frac{1-\gamma}{2 \gamma-1}\right|$, we obtain

$$
\left[F^{\prime \prime}(R)\right]^{2}-A F^{\prime}(R)=0
$$

The general solution of such an equation is a polynomial function [38].

Considering Model II given by (11), we obtain

$$
\left[1-\frac{2 p R\left(\frac{R^{2}}{R_{c}^{2}}+1\right)^{-p-1} \lambda}{R_{c}}\right]\left|\frac{\gamma-1}{2 \gamma-1}\right|-\frac{4 p^{2}\left(\frac{R^{2}}{R_{c}^{2}}+1\right)^{-2 p} R_{c}^{2}\left(R_{c}^{2}-(2 p+1) R^{2}\right)^{2} \lambda^{2}}{\left(R^{2}+R_{c}^{2}\right)^{4}}=0 .
$$

Our issue is now to find the values of $\lambda, p$, and $R / R_{c}$ for which the Solar System experimental constraints are satisfied. Some preliminary considerations are in order at this point. Considering the de Sitter solution achieved from (11), we have $R=$ const $=R_{1}=x_{1} R_{c}$, and $x_{1}>0$. It is straightforward to obtain

$$
\lambda=\frac{x_{1}\left(1+x_{1}^{2}\right)^{p+1}}{2\left[\left(1+x_{1}^{2}\right)^{p+1}-1-(p+1) x_{1}^{2}\right]}
$$

On the other hand, the stability conditions $F_{, R}>0$ and $F_{, R R}>0$ give the inequality

$$
\left(1+x_{1}^{2}\right)^{p+2}>1+(p+2) x_{1}^{2}+(p+1)(2 p+1) x_{1}^{4},
$$

which has to be satisfied. In particular, for $p=1$, it is $x_{1}>\sqrt{3}$ and then $\lambda>\frac{8}{3 \sqrt{3}}=1.5396$. In addition, the value of $x_{1}$ satisfying the relation (86) is also the point where $\lambda\left(x_{1}\right)$, in Eq.(85), reaches its minimum.

To determine values of $R$ compatible with PPN constraints, let us consider the trace of the field equations (2) and explicit solutions, given the density profile $\rho(r)$, in the Solar vicinity. One can set the boundary condition considering $F_{, R_{\infty}}=F_{R_{g}}$

$$
F_{, R_{g}}=F_{, R}\left(R=k^{2} \rho_{g}\right)
$$

where $\rho_{g} \sim 10^{-24} \mathrm{~g} / \mathrm{cm}^{3}$ is the observed Galactic density in the Solar neighborhoods. At this point, we can see when the relation (84) satisfies the constraints for very Long Baseline Interferometer $\left(\gamma-1=4 \times 10^{-4}\right)$ and Cassini Spacecraft $\left(\gamma-1=2.1 \times 10^{-} 5\right)$. This allows to find out suitable values for $p$.

An important remark is in order at this point. These constraint equations work if stability conditions hold. In the range

$$
0<\frac{R}{R_{c}}<\frac{1}{\sqrt{2 p+1}}
$$

$F_{, R R}$ is negative for the model (11) and then stability conditions are violated. To avoid this range, we need, at least, $\frac{R}{R_{c}}>1$. For example, we can choose $\frac{R}{R_{c}}=3.38$, corresponding to de Sitter behavior. Then we have $p=1$ and $\lambda=2$. On the other hand, for $0.944<\lambda<0.966$, we have $p=2$ and $\frac{R}{R_{c}}=\sqrt{3}$; finally, for $R>>R_{c}$, we have $\lambda=2$ and $p=1.5$. For these values of parameters, the Solar System tests are evaded.

Let us consider now Model I, given by (9). Inserting it into the relation (83), we get 


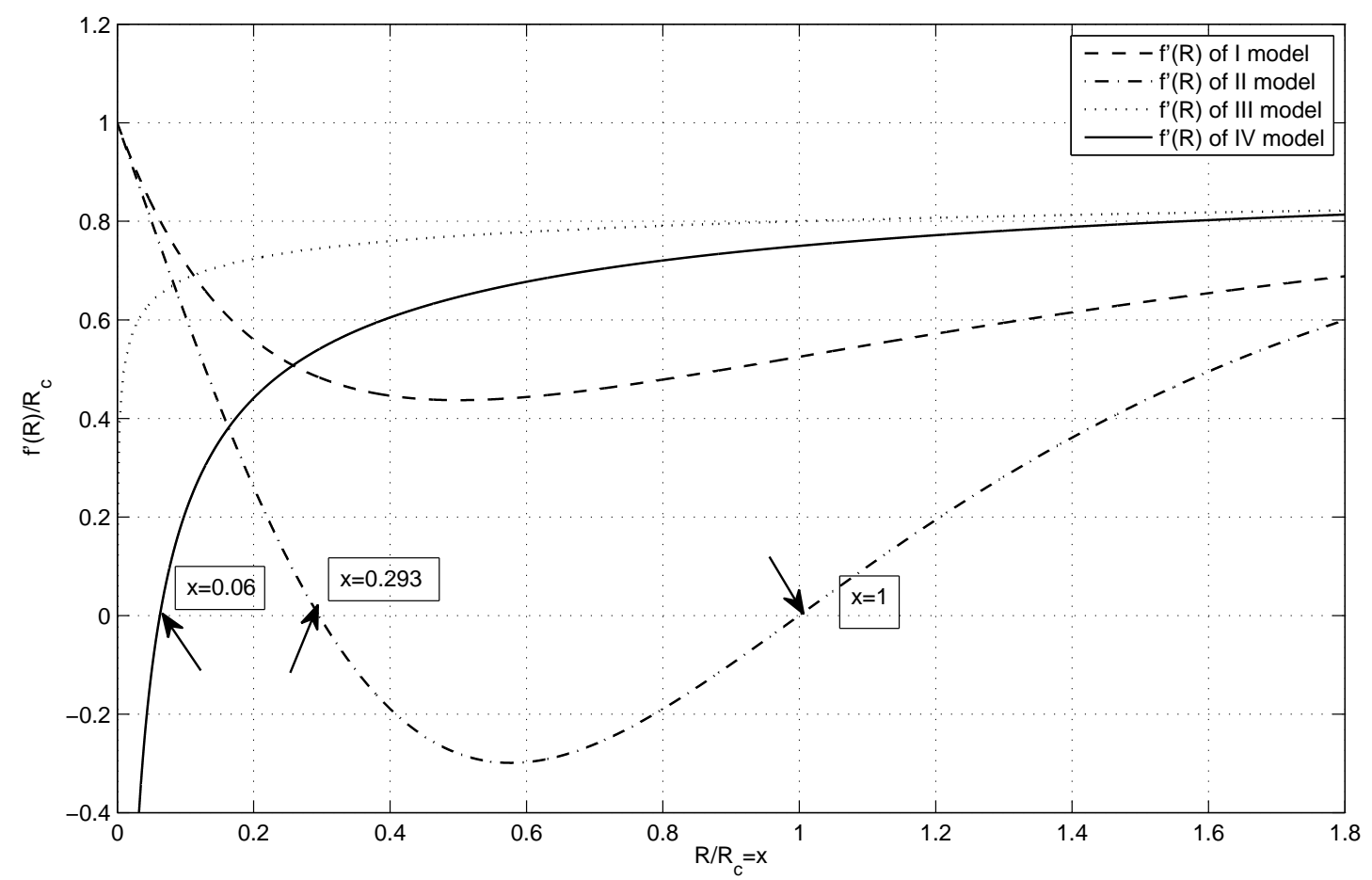

Figure 4: Plots of the first derivatives of four different models as function of $x=\frac{R}{R_{c}}$. Model I (dashed) is drawn for $n=1$ and $\lambda=2$. Model II (dashdot), for $p=2, \lambda=0.95$. Model III (dotted), for $s=0.5$ and $\lambda=1.5$. Model IV (solid) is for $q=0.5$ and $\lambda=0.5$. The labelled values of $x$ indicate where the derivative changes its sign.

$$
\frac{R^{3}\left[\left(\frac{R}{R_{c}}\right)^{2 n}+1\right]^{4}\left[R\left(\left(\frac{R}{R_{c}}\right)^{2 n}+1\right)^{2}-2 n\left(\frac{R}{R_{c}}\right)^{2 n} R_{c} \lambda\right]\left|\frac{\gamma-1}{2 \gamma-1}\right|-4 n^{2}\left[(2 n+1)\left(\frac{R}{R_{c}}\right)^{2 n}-2 n+1\right]^{2}\left(\frac{R}{R_{c}}\right)^{4 n} R_{c}^{2} \lambda^{2}}{R^{4}\left[\left(\frac{R}{R_{c}}\right)^{2 n}+1\right]^{6}}=0
$$

Using the same procedure as above, $\lambda$ is related to the de Sitter behavior. This means

$$
\lambda=\frac{\left(1+x_{1}^{2 n}\right)^{2}}{x_{1}^{2 n-1}\left(2+2 x_{1}^{2 n}-2 n\right)},
$$

while, from the stability conditions, we get

$$
2 x_{1}^{4}-(2 n-1)(2 n+4) x_{1}^{2 n}+(2 n-1)(2 n-2) \geq 0 .
$$

For $n=1$, one obtains $x_{1}>\sqrt{3}, \lambda>\frac{8}{3 \sqrt{3}}$. In this model, $F_{, R R}$ is negative for

$$
0<\frac{R}{R_{c}}<\left(\frac{2 n-1}{2 n+1}\right)^{\frac{1}{2 n}} .
$$

The VLBI constraint is satisfied for $n=1$ and $\lambda=2$, while, for $n=1$ and $\lambda=1.5$, Cassini constraint holds.

By inserting Model III, given by Eq.(12), into the relation (83), we obtain

$$
\frac{R^{3}\left[R-2 s R_{c}\left(\frac{R_{c}}{R}\right)^{2 s} \lambda\right]\left|\frac{\gamma-1}{2 \gamma-1}\right|-4\left(2 s^{2}+s\right)^{2} R_{c}^{2}\left(\frac{R_{c}}{r}\right)^{4 s} \lambda^{2}}{R^{4}}=0 .
$$




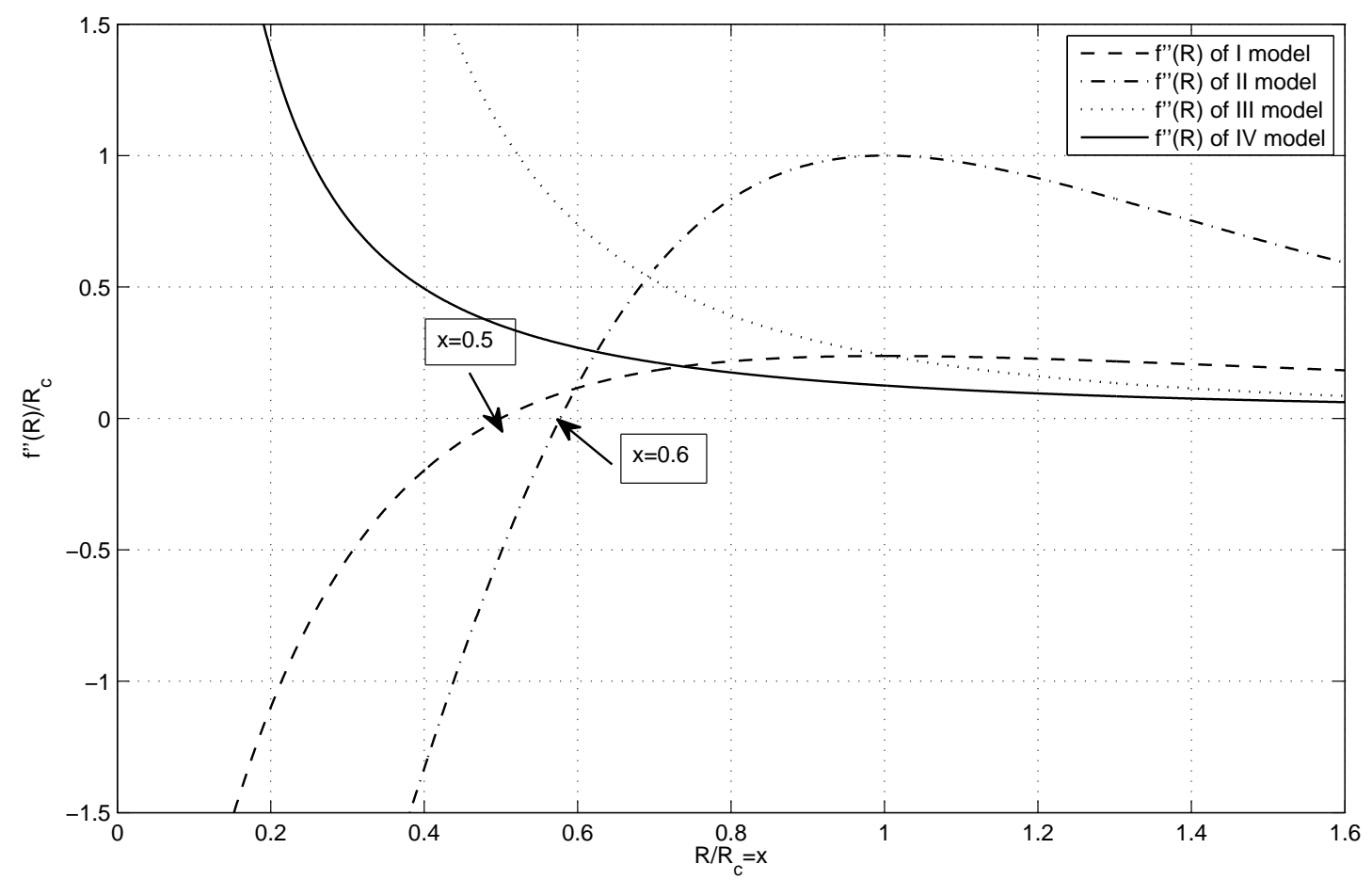

Figure 5: As above for the second derivatives of the models.

The de-Sitter point corresponds to

$$
\lambda=\frac{x_{1}^{2 s+1}}{2\left(x_{1}^{2 s}-s-1\right)} .
$$

while the stability condition is $x_{1}^{2 s}>2 s^{2}+3 s+1$. VLBI and Cassini constraints are satisfied by the sets of values: $s=1, \lambda=1.53$, for $\frac{R}{R_{c}} \sim 1 ; s=2, \lambda=0.95$, for $\frac{R}{R_{c}}=\sqrt{3}, ; s=1, \lambda=2$, for $\frac{R}{R_{c}}=3.38$.

Finally let us consider Model VI, given by Eq.(45), and Model VII, given by Eq.(51). Using Eq.(83) for (45), we get

$$
-\frac{1}{4} b \alpha \operatorname{sech}^{2}\left(\frac{1}{2} b\left(R-R_{0}\right)\right)\left[b^{3} \alpha \operatorname{sech}^{2}\left(\frac{1}{2} b\left(R-R_{0}\right)\right) \tanh ^{2}\left(\frac{1}{2} b\left(R-R_{0}\right)\right)-2\left|\frac{\gamma-1}{2 \gamma-1}\right|\right]=0 .
$$

As above, considering the stability conditions and the de Sitter behavior, we get the parameter ranges $0<b<2$ and $0<\alpha \leq 2$ which satisfy both VLBI and Cassini constraints. Inserting now Model VII in (83), we have

$$
\begin{aligned}
& \frac{1}{2}\left|\frac{\gamma-1}{2 \gamma-1}\right|\left[b \alpha \operatorname{sech}^{2}\left(\frac{1}{2} b\left(R-R_{0}\right)\right)-b_{I} \alpha_{I} \operatorname{sech}^{2}\left(\frac{1}{2} b_{I}\left(R-R_{I}\right)\right)+2\right] \\
& -\frac{1}{4}\left[b^{2} \alpha \operatorname{sech}^{2}\left(\frac{1}{2} b\left(R-R_{0}\right)\right) \tanh \left(\frac{1}{2} b\left(R-R_{0}\right)\right)-b_{I}^{2} \alpha_{I} \operatorname{sech}^{2}\left(\frac{1}{2} b_{I}\left(R-R_{I}\right)\right) \tanh \left(\frac{1}{2} b_{I}\left(R-R_{I}\right)\right)\right]^{2}=0 .
\end{aligned}
$$

From the stability condition, we have that $F_{, R}>0$ for $R>0$, (see Fig 6 ) and $F_{, R R}<0$ for $0<R<2.35$ in suitable units (see Fig [7). Observational constraints from VLBI and Cassini experiments are fulfilled for

$$
R_{I} \gg R_{0}, \quad \alpha_{I} \gg \alpha, \quad b_{I} \ll b .
$$

Plots for $b=2, b_{I}=0.5, \alpha=1.5$ and $\alpha_{I}=2$, verifying the constraints, are reported in Figs. 6 and 7 


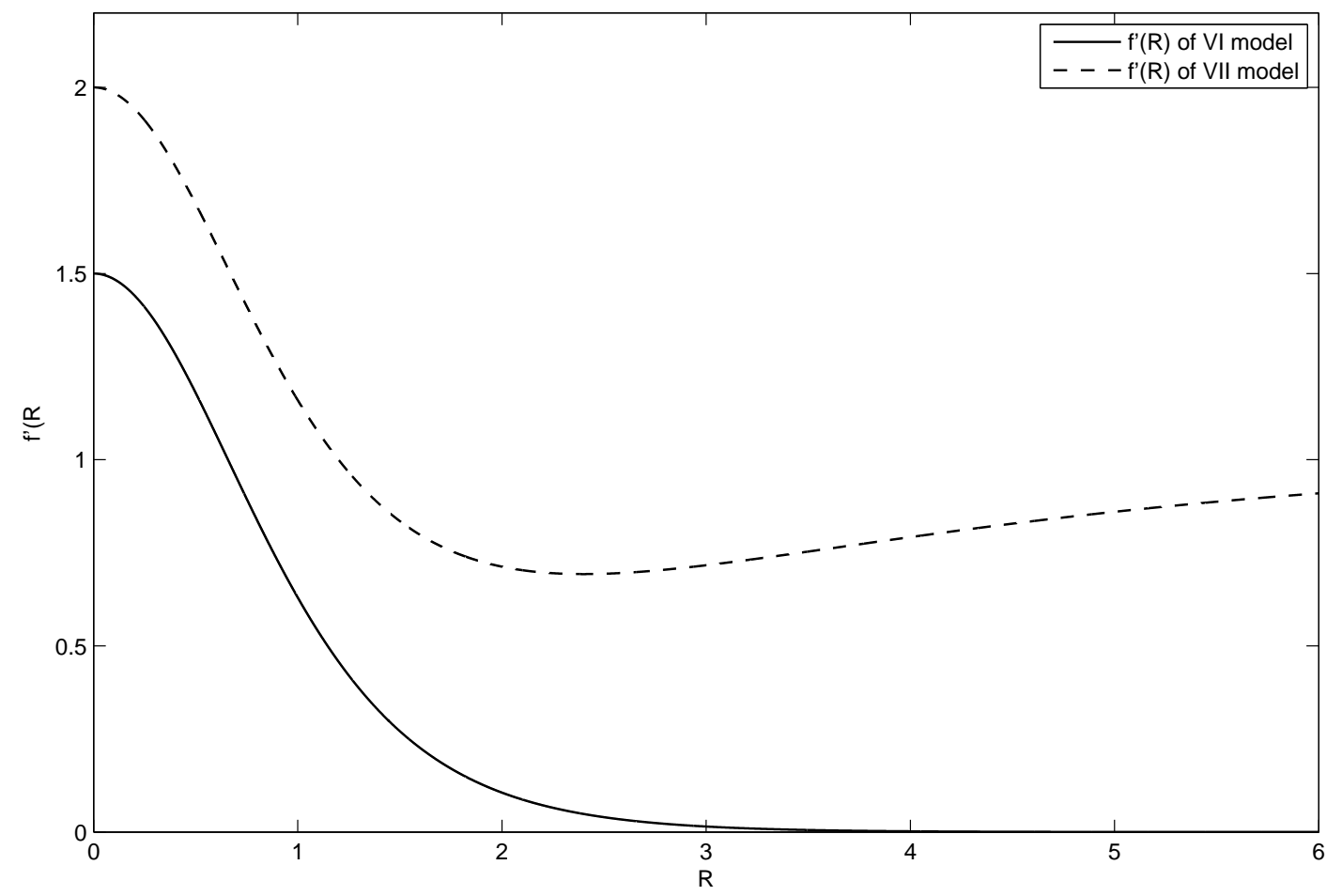

Figure 6: Plots represent the first derivatives of functions (50) (solid line) and (51) (dashed line). Here, $b=2, b_{I}=0.5, \alpha=1.5$ and $\alpha_{I}=2$ with $R_{I}$ with the Solar System value and $R_{0}$ the today cosmological value. It is $F, R>0$ for $R>0$.

Considering now the relation for $\beta$ given by Eq. (82), one can easily verify that it is

$$
\frac{d \gamma}{d R}=-\frac{d}{d R}\left[\frac{F^{\prime \prime}(R)^{2}}{F^{\prime}(R)+2 F^{\prime \prime}(R)^{2}}\right]=0
$$

and this result implies

$$
4(\beta-1)=0 .
$$

This means the complete compatibility of the $f(R)$ solutions between the PPN-parameters $\beta$ and $\gamma$.

Now we want to see if the parameter values, obtained for these models, are compatible with bounds coming from the stochastic background of GWs achieved by interferometric experiments.

\section{STOCHASTIC BACKGROUNDS OF GRAVITATIONAL WAVES TO CONSTRAIN $f(R)$-GRAVITY}

As we said before, also the stochastic background of GWs can be taken into account in order to constrain models. This approach could reveal very interesting because production of primordial GWs could be a robust prediction for any model attempting to describe the cosmological evolution at primordial epochs. However, bursts of gravitational radiation emitted from a large number of unresolved and uncorrelated astrophysical sources generate a stochastic background at more recent epochs, immediately following the onset of galaxy formation. Thus, astrophysical backgrounds might overwhelm the primordial one and their investigation provides important constraints on the signal detectability coming from the very early Universe, up to the bounds of the Planck epoch and the initial singularity [45, 54, 55, 57]. 


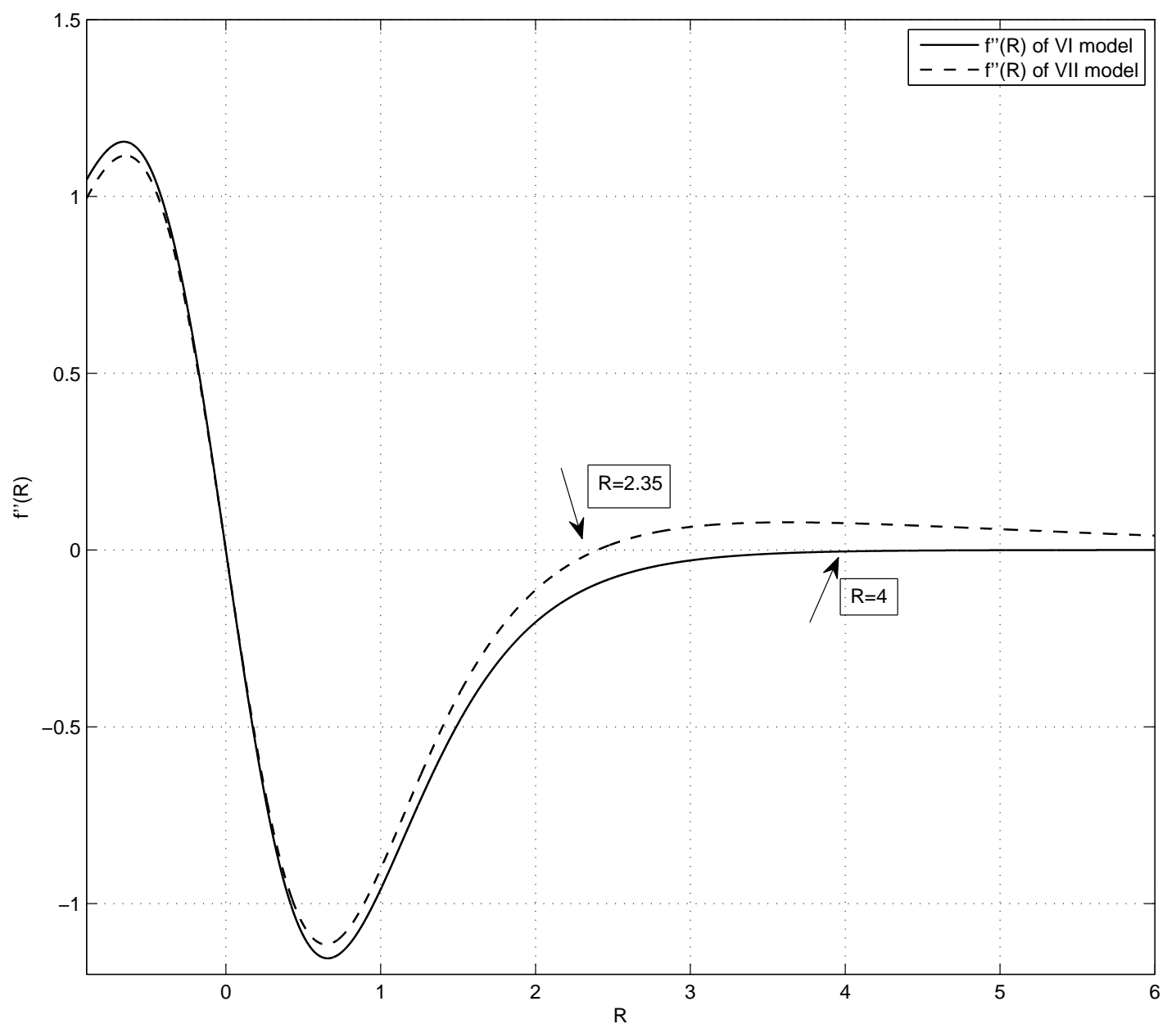

Figure 7: Second derivatives of Model VI (solid line) and VII (dashed line). Here $F_{, R R}$ is negative in the range $0<R<4$ for Model VI and in the range $0<R<2.35$ for Model VII. As above, we have used $b=2, b_{I}=0.5, \alpha=1.5$ and $\alpha_{I}=2$ with the value of $R_{I}$ taken in the Solar System and $R_{0}$ for the today cosmological value.

It is worth stressing the unavoidable and fundamental character of such a mechanism. It directly derives from the inflationary scenario [58, 59], which well fits the WMAP data with particular good agreement with almost exponential inflation and spectral index $\approx 1,[60,61]$.

The main characteristics of the gravitational backgrounds produced by cosmological sources depend both on the emission properties of each single source and on the source rate evolution with redshift. It is therefore interesting to compare and contrast the probing power of these classes of $f(R)$-models at hight, intermediate and zero redshift [62].

To this purpose, let us take into account the primordial physical process which gave rise to a characteristic spectrum $\Omega_{\text {sgw }}$ for the early stochastic background of relic scalar GWs by which we can recast the further degrees of freedom coming from fourth-order gravity. This approach can greatly contribute to constrain viable cosmological models. The physical process related to the production has been analyzed, for example, in [54, 55, 56] but only for the first two tensorial components due to standard General Relativity. Actually the process can be improved considering also the third scalar-tensor component strictly related to the further $f(R)$ degrees of freedom [47].

Before starting with the analysis, it has to be emphasized that the stochastic background of scalar GWs can be described in terms of a scalar field $\Phi$ and characterized by a dimensionless spectrum (see the analogous definitions for tensorial waves in $[45,54,55,57])$. We can write the energy density of scalar GWs in terms of the closure energy 
density of GWs per logarithmic frequency interval as

$$
\Omega_{s g w}(f)=\frac{1}{\rho_{c}} \frac{d \rho_{s g w}}{d \ln f},
$$

where

$$
\rho_{c} \equiv \frac{3 H_{0}^{2}}{8 \pi G}
$$

is the critical energy density of the Universe, $H_{0}$ the today observed Hubble expansion rate, and $d \rho_{\text {sgw }}$ is the energy density of the gravitational radiation scalar part contained in the frequency range from $f$ to $f+d f$. We are considering now standard units.

The calculation for a simple inflationary model can be performed assuming that the early Universe is described by an inflationary de Sitter phase emerging in the radiation dominated era [54, 55, 57]. The conformal metric element is

$$
d s^{2}=a^{2}(\eta)\left[-d \eta^{2}+d \vec{x}^{2}+h_{\mu \nu}(\eta, \vec{x}) d x^{\mu} d x^{\nu}\right],
$$

and a GW with tensor and scalar modes in the $z+$ direction is given by [47]

$$
\tilde{h}_{\mu \nu}(t-z)=A^{+}(t-z) e_{\mu \nu}^{(+)}+A^{\times}(t-z) e_{\mu \nu}^{(\times)}+\Phi(t-z) e_{\mu \nu}^{(s)} .
$$

The pure scalar component is then

$$
h_{\mu \nu}=\Phi e_{\mu \nu}^{(s)},
$$

where $e_{\mu \nu}^{(s)}$ is the polarization tensor.

It is possible to write an expression for the energy density of the stochastic relic scalar gravitons in the frequency interval $(\omega, \omega+d \omega)$ as

$$
d \rho_{s g w}=2 \hbar \omega\left(\frac{\omega^{2} d \omega}{2 \pi^{2} c^{3}}\right) N_{\omega}=\frac{\hbar H_{d s}^{2} H_{0}^{2}}{4 \pi^{2} c^{3}} \frac{d \omega}{\omega}=\frac{\hbar H_{d s}^{2} H_{0}^{2}}{4 \pi^{2} c^{3}} \frac{d f}{f},
$$

where $f$, as above, is the frequency in the standard comoving time. Eq.(105) can be written in terms of the today and de Sitter values of energy density being

$$
H_{0}=\frac{8 \pi G \rho_{c}}{3 c^{2}}, \quad H_{d s}=\frac{8 \pi G \rho_{d s}}{3 c^{2}} .
$$

Introducing the Planck density $\rho_{\text {Planck }}=\frac{c^{7}}{\hbar G^{2}}$, the spectrum is given by

$$
\Omega_{s g w}(f)=\frac{1}{\rho_{c}} \frac{d \rho_{s g w}}{d \ln f}=\frac{f}{\rho_{c}} \frac{d \rho_{s g w}}{d f}=\frac{16}{9} \frac{\rho_{d s}}{\rho_{\text {Planck }}} .
$$

At this point, some comments are in order. First of all, such a calculation works for a simplified model which does not include the matter dominated era. If such an era is also included, the redshift at equivalence epoch has to be considered. Taking into account also results in [56], we get

$$
\Omega_{\text {sgw }}(f)=\frac{16}{9} \frac{\rho_{d s}}{\rho_{\text {Planck }}}\left(1+z_{e q}\right)^{-1},
$$

for the waves which, at the epoch in which the Universe becomes matter dominated, have a frequency higher than $H_{e q}$, the Hubble parameter at equivalence. This situation corresponds to frequencies $f>\left(1+z_{e q}\right)^{1 / 2} H_{0}$. The redshift correction in Eq.(108) is needed since the today observed Hubble parameter $H_{0}$ would result different without a matter dominated contribution. At lower frequencies, the spectrum is given by [54, 55]

$$
\Omega_{\text {sgw }}(f) \propto f^{-2} .
$$

Nevertheless, since the spectrum falls off $\propto f^{-2}$ at low frequencies, this means that today, at LIGO-VIRGO and LISA frequencies (indicated in Fig. 8), one gets

$$
\Omega_{\text {sgw }}(f) h_{100}^{2}<2.3 \times 10^{-12} .
$$




\begin{tabular}{|l|c|}
\hline$\Phi_{c}(100 H z)<2 \times 10^{-26}$ & LIGO \\
\hline$\Phi_{c}(100 H z)<2 \times 10^{-25}$ & VIRGO \\
\hline$\Phi_{c}(100 H z)<2 \times 10^{-21}$ & LISA \\
\hline
\end{tabular}

Table II: Upper limits on the expected amplitude for the GW scalar component.

It is interesting to calculate the corresponding strain at $\approx 100 \mathrm{~Hz}$, where interferometers like VIRGO and LIGO reach a maximum in sensitivity (see e.g. [63, 64]). The well known equation for the characteristic amplitude [54, 55], adapted to the scalar component of GWs, can be used. It is

$$
\Phi_{c}(f) \simeq 1.26 \times 10^{-18}\left(\frac{1 H z}{f}\right) \sqrt{h_{100}^{2} \Omega_{s g w}(f)}
$$

and then we obtain the values in the Table II

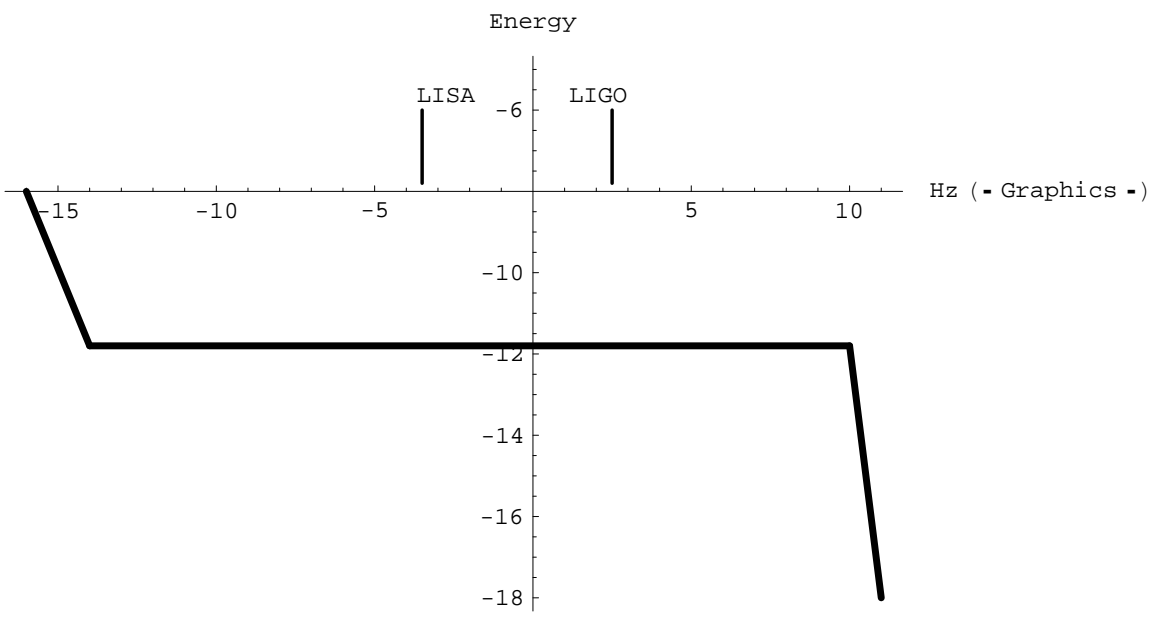

Figure 8: The spectrum of relic scalar GWs in inflationary models is flat over a wide range of frequencies. The horizontal axis is $\log _{10}$ of frequency, in $\mathrm{Hz}$. The vertical axis is $\log _{10} \Omega_{g s w}$. The inflationary spectrum rises quickly at low frequencies (wave which re-entered in the Hubble sphere after the Universe became matter dominated) and falls off above the (appropriately redshifted) frequency scale $f_{\max }$ associated with the fastest characteristic time of the phase transition at the end of inflation. The amplitude of the flat region depends only on the energy density during the inflationary stage; we have chosen the largest amplitude consistent with the WMAP constrains on scalar perturbations. This means that, at LIGO and LISA frequencies, we have $\Omega_{\text {sgw }}<2.3 * 10^{-12}$

In summary, the above results point out that a further scalar component of GWs, coming e.g. from $f(R)$-gravity, should be seriously considered in the signal detection of interferometers. As discussed in [62], this fact could constitute either an independent test for alternative theories of gravity or a further probe of GR capable of ruling out other theories.

At this point, using the above LIGO, VIRGO and LISA upper bounds, calculated for the characteristic amplitude of GW scalar component, let us test the $f(R)$-gravity models, considered in the previous sections, to see whether they are compatible both with the Solar System and GW stochastic background. 
Before starting with the analysis, taking into account the discussion in Sec.II, we have that the GW scalar component is derived considering

$$
\Phi=-\frac{\delta \sigma}{\sigma_{0}}, \quad \sigma=-\ln \left(1+f^{\prime}(A)\right)=\ln F^{\prime}(A), \quad \delta \sigma=\frac{f^{\prime \prime}(A)}{1+f^{\prime}(A)} \delta A
$$

As standard, we are assuming small perturbations in the conformal frame [47]). This means

$$
g_{\mu \nu}=\eta_{\mu \nu}+h_{\mu \nu}, \quad \sigma=\sigma_{0}+\delta \sigma
$$

These assumptions allow to derive the "linearized" curvature invariants $\widetilde{R}_{\mu \nu \rho \sigma}, \widetilde{R}_{\mu \nu}$ and $\widetilde{R}$ and then the linearized field equations [53]

$$
\begin{gathered}
\widetilde{R}_{\mu \nu}-\frac{\widetilde{R}}{2} \eta_{\mu \nu}=-\partial_{\mu} \partial_{\nu} \Phi+\eta_{\mu \nu} \square \Phi \\
\square \Phi=m^{2} \Phi
\end{gathered}
$$

As above, for the considered models, we have to determine the values of the characteristic parameters which are compatible with both Solar System and GW stochastic background.

Let us start, for example, with the model (12). Starting from the definitions (112), it is straightforward to derive the scalar component amplitude

$$
\Phi_{I I I}=\frac{s(2 s+1)\left(\frac{R_{c}}{R}\right)^{2 s+1} \lambda}{\left[s R_{c}\left(\frac{R_{c}}{R}\right)^{2 s} \lambda-R\right] \log \left[2-2 s\left(\frac{R_{c}}{R}\right)^{2 s+1} \lambda\right]} .
$$

Such an equation satisfies the constraints in Table $\leqq$ for the values $s=0.5, \frac{R}{R_{c}} \sim 1, \lambda=1.53$ and $s=1, \frac{R}{R_{c}} \sim 1$, $\lambda=0.95(\mathrm{LIGO}) ; s=2, \frac{R}{R_{c}}=\sqrt{3}, \lambda=2(\mathrm{VIRGO}) ; s=1, \lambda=2$ and $\frac{R}{R_{c}}=3.38$ (LISA).

It is important to stress the nice agreement with the figures achieved from the PPN constraints. In this case, we have assumed $R_{c} \sim \rho_{c} \sim 10^{-29} \mathrm{~g} / \mathrm{cm}^{3}$, where $\rho_{c}$ is the present day cosmological density.

Considering the model (9), we obtain

$$
\Phi_{I}=-\frac{n\left[(2 n+1)\left(\frac{R}{R_{c}}\right)^{2 n}-2 n+1\right]\left(\frac{R}{R_{c}}\right)^{2 n-1} \lambda}{\left[\left(\frac{R}{R_{c}}\right)^{2 n}+1\right]\left\{R\left[\left(\frac{R}{R_{c}}\right)^{2 n}+1\right]^{2}-n\left(\frac{R}{R_{c}}\right)^{2 n} R_{c} \lambda\right\} \log \left(1-\frac{2 n\left(\frac{R}{R_{c}}\right)^{2 n-1} \lambda}{\left(\left(\frac{R}{R_{c}}\right)^{2 n}+1\right)^{2}}\right)} .
$$

The expected constraints for GW scalar amplitude are fulfilled for $n=1$ and $\lambda=2$ and for $n=1$ and $\lambda=1.5$ when $0.3<\frac{R}{R_{c}}<1$.

Furthermore, considering the model (11), one gets

$$
\Phi_{I}=-\frac{2 p\left(1+\frac{R^{2}}{R_{c}^{2}}\right)^{-p} R_{c}\left((1+2 p) R^{2}-R_{c}^{2}\right) \lambda}{\left(R^{2}-R_{c}^{2}\right)^{2}\left[2-\frac{2 p\left(1+\frac{R^{2}}{R_{c}^{2}}\right)^{-1-p} \lambda}{R_{c}}\right] \ln \left[2-\frac{2 p R\left(1+\frac{R^{2}}{R_{c}^{2}}\right)^{-1-p} \lambda}{R_{c}}\right]} .
$$

The LIGO upper bound is fulfilled for $p=1, \frac{R}{R_{c}}>\sqrt{3}, \lambda>\frac{8}{3 \sqrt{3}}$; the VIRGO one for $p=1, \frac{R}{R_{c}}=3.38, \lambda=2$; finally, for LISA, we have $p=2, \frac{R}{R_{c}}=\sqrt{3}$ and $0.944<\lambda<0.966$. Besides, considering LISA in the regime $R>>R_{c}$, we have $\lambda=2$ and $p=1.5$.

Finally, let us consider Models VI and VII. We have

$$
\Phi_{V I}=\frac{b^{2} \alpha \tanh \left[\frac{1}{2} b\left(R-R_{0}\right)\right]}{\left[b \alpha+\cosh \left(b\left(R-R_{0}\right)\right)+1\right] \ln \left[\frac{b \alpha}{\cosh \left(b\left(R-R_{0}\right)\right)+1}\right]}
$$


and

$$
\begin{aligned}
\Phi_{V I I}= & \log \left[0.5\left(b \alpha \operatorname{sech}^{2}\left(0.5 b\left(R-R_{0}\right)\right)-b_{I} \alpha_{I} \operatorname{sech}^{2}\left(0.5 b_{I}\left(R-R_{I}\right)\right)+2\right)\right] \\
& \times\left[b \alpha \operatorname{sech}^{2}\left(0.5 b\left(R-R_{0}\right)\right)-b_{I} \alpha_{I} \operatorname{sech}^{2}\left(0.5 b_{I}\left(R-R_{I}\right)\right)+4\right] \\
& \times\left[b^{2} \alpha \operatorname{sech}^{2}\left(0.5 b\left(R-R_{0}\right)\right) \tanh \left(0.5 b\left(R-R_{0}\right)\right)-b_{I}^{2} \alpha_{I} \operatorname{sech}^{2}\left(0.5 b_{I}\left(R-R_{I}\right)\right) \tanh \left(0.5 b_{I}\left(R-R_{I}\right)\right)\right] .
\end{aligned}
$$

These equations satisfy the constraints for VIRGO, LIGO and LISA for $b=2, b_{I}=0.5, \alpha=1.5$ and $\alpha_{I}=2$ with $R_{I}$ valued at Solar System scale and $R_{0}$ at cosmological scale.

\section{CONCLUSIONS}

In this paper, we have investigated the possibility that some viable $f(R)$ models could be constrained considering both Solar System experiments and upper bounds on the stochastic background of gravitational radiation. Such bounds come from interferometric ground-based (VIRGO and LIGO) and space (LISA) experiments. The underlying philosophy is to show that the $f(R)$ approach, in order to describe consistently the observed universe, should be tested at very different scales, that is at very different redshifts. In other words, such a proposal could partially contribute to remove the unpleasant degeneracy affecting the wide class of dark energy models, today on the ground.

Beside the request to evade the Solar System tests, new methods have been recently proposed to investigate the evolution and the power spectrum of cosmological perturbations in $f(R)$ models [24]. The investigation of stochastic background, in particular of the scalar component of GWs coming from the $f(R)$ additional degrees of freedom, could acquire, if revealed by the running and forthcoming experiments, a fundamental importance to discriminate among the various gravity theories [62]. These data (today only upper bounds coming from simulations) if combined with Solar System tests, CMBR anisotropies, LSS, etc. could greatly help to achieve a self-consistent cosmology bypassing the shortcomings of $\Lambda \mathrm{CDM}$ model.

Specifically, we have taken into account some broken power law $f(R)$ models fulfilling the main cosmological requirements which are to match the today observed accelerated expansion and the correct behavior in early epochs. In principle, the adopted parameterization allows to fit data at extragalactic and cosmological scales [16]. Furthermore, such models are constructed to evade the Solar System experimental tests. Beside these broken power laws, we have considered also two models capable of reproducing the effective cosmological constant, the early inflation and the late acceleration epochs [20]. These $f(R)$-functions are combinations of hyperbolic tangents.

We have discussed the behavior of all the considered models. In particular, the problem of stability has been addressed determining suitable and physically consistent ranges of parameters. Then we have taken into account the results of the main Solar System current experiments. Such results give upper limits on the PPN parameters which any self-consistent theory of gravity should satisfy at local scales. Starting from these, we have selected the $f(R)$ parameters fulfilling the tests. As a general remark, all the functional forms chosen for $f(R)$ present sets of parameters capable of matching the two main PPN quantities, that is $\gamma_{e x p}$ and $\beta_{\text {exp }}$. This means that, in principle, extensions of GR are not a priori excluded as reasonable candidates for gravity theories. To construct such extensions, the reconstruction method developed in [65] may be applied.

The interesting feature, and the main result of this paper, is that such sets of parameters are not in conflict with bounds coming from the cosmological stochastic background of GWs. In particular, some sets of parameters reproduce quite well both the PPN upper limits and the constraints on the scalar component amplitude of GWs.

Far to be definitive, these preliminary results indicate that self-consistent models could be achieved comparing experimental data at very different scales without extrapolating results obtained only at a given scale.

\section{ACKNOWLEDGEMENTS}

This research is supported by INFN-CSIC bilateral project, by Azione Integrata Italia-Spagna 2007 (MIUR Prot. No. 464, 13/3/2006) grant and by MCIN (Spain) projects FIS2006-02842 and PIE2007-50I023. The work by S.N. is supported by Min. of Education, Science, Sports and Culture of Japan under grant no. 18549001.

[1] P.J.E. Peebles and B. Ratra Rev. Mod. Phys. 75, 559 (2003).

[2] S. Capozziello, V.F. Cardone and A. Troisi Jou. Cosm. and Astrop. Phys. 08, 001 (2006). 
[3] A. Vecchiato, M. G. Lattanzi, B. Bucciarelli, M. T. Crosta, F. de Felice and M. Gai, Astron. Astrophys. 399, 337 (2003). [4] V. Faraoni, Phys. Rev. D 72, 124005 (2005); G. Cognola and S. Zerbini, J. Phys. A 39, 6245 (2006).

[5] K. S. Stelle, Gen. Rel. Grav. 9, 353 (1978).

[6] S. Nojiri and S. D. Odintsov, Int. J. Geom. Meth. Mod. Phys. 4, 115 (2007), hep-th/0601213, arXiv:0807.0685[hep-th].

[7] S. Capozziello and M. Francaviglia, Gen. Rel. Grav.: Special Issue on Dark Energy 40, 357 (2008).

[8] T. P. Sotiriou and V. Faraoni, arXiv:0805.1726 [gr - qc] (2008).

[9] S. Capozziello, Int. J. Mod. Phys. D 11, 483, (2002); S. Capozziello, S. Carloni and A. Troisi, Rec. Res. Develop. Astron. Astrophys. 1, 625 (2003), arXiv:astro - ph/0303041; S. Capozziello, V. F. Cardone, S. Carloni and A. Troisi, Int. J. Mod. Phys. D, 12, 1969 (2003).

[10] S. Nojiri and S. D. Odintsov, Phys. Lett. B 576, 5, (2003), hep-th/0307071, Phys. Rev. D 68, 123512, (2003), hep-th/0307288, Gen. Rel. Grav. 36, 1765, (2004), hep-th/0308176, S. M. Carroll, V. Duvvuri, M. Trodden and M. S. Turner, Phys. Rev. D 70, 043528 (2004); G. Allemandi, A. Borowiec and M. Francaviglia, Phys. Rev. D 70, 103503 (2004).

[11] S. Capozziello, V. F. Cardone and A. Troisi, Phys. Rev. D 71, 043503 (2005).

[12] S. Capozziello, P. Martin - Moruno, C. Rubano, Phys. Lett. B 664, 12 (2008); S. Capozziello, S. Nojiri, S.D. Odintsov and A. Troisi, Phys. Lett. B639,135 (2006), astro-ph/0604431

[13] A. A. Starobinsky, JETP Lett. 86, 157 (2007).

[14] B. Li and J. D. Barrow, Phys. Rev. D 75, 084010 (2007).

[15] J. Khoury and A. Weltman, Phys. Rev. Lett. 93, 171104 (2004); Phys. Rev. D 69, 044026 (2004); S. Capozziello and S. Tsujikawa, Phys. Rev. D 77, 107501 (2008); N. Deruelle, M. Sasaki, Y. Sendouda, Phys. Rev. D 77, 124024 (2008); H.-J. Schmidt, arXiv:0803.0920 gr-qc] (2008).

[16] W. Hu and I. Sawicki, Phys. Rev. D 76064004 (2007).

[17] S. A. Appleby and R. A. Battye, Phys. Lett. B 654, 7 (2007).

[18] S. Tsujikawa, arXiv:0709.1391 [astro-ph], to appear in Physical Review D.

[19] S. Nojiri and S. D. Odintsov, Phys. Rev. D 77, 026007 (2008) [arXiv:0710.1738 [hep-th]].

[20] G. Cognola, E. Elizalde, S. Nojiri, S.D. Odintsov, L. Sebastiani and S.Zerbini, Phys. Rev. D77, 046009 (2008), arXiv:0712.4017 [hep-th].

[21] S. Nojiri and S.D. Odintsov, Phys. Lett. B 657, 238 (2008), arXiv:0707.1941[hep-th].

[22] M. Amarzguioui, O. Elgaroy, D. F. Mota and T. Multamaki, Astron. Astrophys. 454, 707 (2006); S. M. Carroll, I. Sawicki, A. Silvestri and M. Trodden, New J. Phys. 8, 323 (2006); R. Bean, D. Bernat, L. Pogosian, A. Silvestri and M. Trodden, Phys. Rev. D 75, 064020 (2007); Y. S. Song, W. Hu and I. Sawicki, Phys. Rev. D 75, 044004 (2007); Y. S. Song, H. Peiris and W. Hu, Phys. Rev. D 76, 063517 (2007); L. Pogosian and A. Silvestri, arXiv:0709.0296 [astro-ph]; A. De Felice, P. Mukherjee and Y. Wang, arXiv:0706.1197 [astro-ph].

[23] T. Faulkner, M. Tegmark, E. F. Bunn and Y. Mao, Phys. Rev. D 76, 063505 (2007).

[24] H. Oyaizu, arXiv:0807.2449 [astro-ph]; H. Oyaizu, M. Lima, W. Hu, arXiv:0807.2462 [astro-ph].

[25] S. Capozziello, V. F. Cardone and A. Troisi, Mon. Not. Roy. Astron. Soc. 375, 1423 (2007).

[26] C. F. Martins and P. Salucci, Mon. Not. Roy. Astron. Soc. 381, 1103 (2007).

[27] Y. Sobouti, arXiv:astro-ph/0603302

[28] S. Mendoza and Y. M. Rosas-Guevara, Astron. Astrophys. 472, 367 (2007).

[29] C. G. Boehmer, T. Harko and F. S. N. Lobo, arXiv:0709.0046 [gr-qc]. S. Nojiri and S.D. Odintsov, arXiv:0801.4843]astro$\mathrm{ph}]$.

[30] F.S.N. Lobo, arXiv: 0807.1640[gr-qc] (2008).

[31] L. Amendola and S. Tsujikawa, arXiv:0705.0396 [astro-ph].

[32] O. Bertolami, C. G. Boehmer, T. Harko and F. S. N. Lobo, Phys. Rev. D 75, 104016 (2007);

[33] S. Capozziello and A. Troisi, Phys. Rev. D 72, 044022 (2005).

[34] G. J. Olmo, Phys. Rev. Lett. 95, 261102 (2005); G. Allemandi, M. Francaviglia, M. L. Ruggiero and A. Tartaglia, Gen. Rel. Grav. 37, 1891 (2005).

[35] I. Navarro and K. Van Acoleyen, JCAP 0702, 022 (2007).

[36] A. L. Erickcek, T. L. Smith and M. Kamionkowski, Phys. Rev. D 74, 121501 (2006); T. Chiba, T. L. Smith and A. L. Erickcek, Phys. Rev. D 75, 124014 (2007).

[37] G. J. Olmo, Phys. Rev. D 72, 083505 (2005); V. Faraoni, Phys. Rev. D 74, 023529 (2006); A. F. Zakharov, A. A. Nucita, F. De Paolis and G. Ingrosso, Phys. Rev. D 74, 107101 (2006); G. Allemandi and M. L. Ruggiero, Gen. Rel. Grav. 39, 1381 (2007); S. Tsujikawa, Phys. Rev. D 76, 023514 (2007); S. Nojiri and S. D. Odintsov, Phys. Lett. B 652, 343 (2007), arXiv:0706.1378 hep-th]; X. H. Jin, D. J. Liu and X. Z. Li, arXiv:astro-ph/0610854 A.Lev et al, arXiv:0807.3445[hep-th].

[38] S. Capozziello, A. Stabile and A. Troisi, Mod. Phys. Lett. A 21, 2291 (2006); Phys. Rev. D 76, 104019 (2007).

[39] T. P. Sotiriou, Gen. Rel. Grav. 38, 1407 (2006).

[40] T. Multamaki and I. Vilja, Phys. Rev. D 74, 064022 (2006); Phys. Rev. D 76, 064021 (2007); arXiv:0709.3422 [astro-ph]; T. Kobayashi and K. Maeda, arXiv:0807.2503 astro-ph].

[41] K. Kainulainen, J. Piilonen, V. Reijonen and D. Sunhede, Phys. Rev. D 76, 024020 (2007),

K. Kainulainen and D. Sunhede, arXiv: 0803.0867 [gr-qc] (2008). G. Cognola, E. Elizalde, S. Nojiri, S.D. Odintsov and S. Zerbini, JCAP 0502, 010(2005), hep-th/0501096

[42] J. Barrow and A.C. Ottewill, J. Phys. A: Math. Gen. 16, 2757 (1983).

[43] S. Capozziello, A. Stabile and A. Troisi, Class. Quant. Grav. 24, 2153 (2007); Class. Quant. Grav. 25, 085004 (2008).

[44] S. Capozziello, A. Stabile, A. Troisi , Phys. Rev. D 76, 104019 (2007). 
[45] M. Maggiore - Phys. Rep. 331, 283-367 (2000).

[46] D. Babusci, L. Baiotti, F. Fucito, A. Nagar, Phys. Rev. D 64, 062001 (2001).

[47] S. Capozziello, C. Corda, M. De Laurentis, Mod. Phys Lett. A 22, 2647 (2007).

[48] A. D. Dolgov and M. Kawasaki, Phys. Lett. B 5731 (2003).

[49] I.I. Shapiro, in General Relativity and Gravitation 12, eds. N. Ashby et al. (cambridge Univ. Press, 1993).

[50] J.G. Williams et al, Phys. Rev. D 53, 6730 (1996).

[51] S.S. Shapiro et al, Phys. Rev. D 92, 121101 (2004).

[52] B. Bertotti, L. Iess and P. Tortora, Nature 425, 374 (2003).

[53] C. W. Misner , K. S. Thorne and J. A. Wheeler - "Gravitation" - W. H. Feeman and Company 1973.

[54] B. Allen -Proceedings of the Les Houches School on Astrophysical Sources of Gravitational Waves, eds. Jean-Alain Marck and Jean-Pierre Lasota (Cambridge University Press, Cambridge, England 1998).

[55] L. Grishchuk et al. - Phys. Usp. 44, 1 (2001); Usp.Fiz.Nauk 171, 3 (2001).

[56] B. Allen - Phys. Rev. D 3, 2078 (1988).

[57] B. Allen and A.C. Ottewill - Phys. Rev. D 56, 545 (1997).

[58] G.S. Watson- "An exposition on inflationary cosmology" - North Carolina University Press (2000).

[59] A. Guth - Phys. Rev. 23, 347 (1981).

[60] C.L. Bennet et al. - ApJS 148, 1 (2003).

[61] D.N. Spergel et al. - ApJS 148, 175, 2003.

[62] S. Capozziello, M. De Laurentis, M. Francaviglia Astrop. Phys. 29, 125 (2008).

[63] http://www.ligo.org/pdf_public/camp.pdf.

[64] http://www.ligo.org/pdf_public/hough02.pdf.

[65] S. Nojiri and S.D. Odintsov, Phys.Rev. D74, 086005 (2006), hep-th/0608008 